\title{
Fixed points on torus fiber bundles over the circle
}

\author{
by \\ D. L. Gonçalves (São Paulo), D. Penteado (São Carlos) and \\ J. P. Vieira (Rio Claro)
}

\begin{abstract}
The main purpose of this work is to study fixed points of fiber-preserving maps over the circle $S^{1}$ for spaces which are fibrations over $S^{1}$ and the fiber is the torus $T$. For the case where the fiber is a surface with nonpositive Euler characteristic, we establish general algebraic conditions, in terms of the fundamental group and the induced homomorphism, for the existence of a deformation of a map over $S^{1}$ to a fixed point free map. For the case where the fiber is a torus, we classify all maps over $S^{1}$ which can be deformed fiberwise to a fixed point free map.
\end{abstract}

\section{INTRODUCTION}

Given a fibration $E \rightarrow B$ and a fiber-preserving map $f: E \rightarrow E$ over $B$, the question if $f$ can be deformed over $B$ to a fixed point free map has been considered by many authors (see for example [Do-74], [F-H-81] and [Go-87]). In [F-H-81], E. Fadell and S. Husseini showed that the above problem can be stated in terms of obstructions (including higher ones). This is obtained under the hypothesis that the base, the total space and the fiber $F$ are manifolds, and the dimension of $F$ is greater than or equal to 3 . The case where the fiber has dimension 2 is not considered. This case, even when the base is a point, is still a main open problem; when the total space is a surface with negative Euler characteristic it is known that the vanishing of the Nielsen number is not equivalent to the existence of a deformation to a fixed point free map (see [Ni-27], [Ji-85] and [Ke-87]).

Consider a fiber-preserving map $f: M \rightarrow M$, where $M$ is a fiber bundle over the circle $S^{1}$ and the fiber is a closed surface $S$. Such fiber bundles are obtained from $S \times[0,1]$ by identifying $(x, 0)$ with $(\phi(x), 1)$, where $\phi$ is a homeomorphism of $S$. The main purpose of this work is to study in detail the case where the fiber is a closed surface. We develop a few generalities when $S \neq S^{2}$ and $S \neq \mathbb{R} P^{2}$. For the cases when the fiber is either the

2000 Mathematics Subject Classification: Primary 55M20; Secondary 55R10.

Key words and phrases: fixed point, fiber bundle, fiberwise homotopy, abelianized obstruction. 
sphere $S^{2}$ or the projective plane $\mathbb{R} P^{2}$ the classical obstruction theory can be used. Then we specialize to the case where the fiber is a torus.

In this latter case we denote the total space by $M A$. We classify the fiber-preserving maps $f: M A \rightarrow M A$ over $S^{1}$, i.e. $p \circ f=p$, which can be deformed to a fixed point free $g$ by a fiberwise homotopy over $S^{1}$. The homotopy class of $f$ is given in terms of the induced homomorphism $f_{\#}$ : $\pi_{1}(M A) \rightarrow \pi_{1}(M A)$ on the fundamental groups. This is Theorem 4.1.

In [Pe-88], for fiber-preserving maps, one defines the abelianized obstruction to deforming a fiber-preserving map over $B$ to a fixed point free map as a cohomology class, and then computes this class on the principal torus bundle. It is shown that, in general, the vanishing of this class is not sufficient to guarantee that the map can be deformed over $S^{1}$ to a fixed point free map. We analyze this question for the fiber bundles $M A \rightarrow S^{1}$ in [G-P-V-03].

This paper is organized into four sections. In Section 1 we consider the general case of a surface bundle over the circle $S^{1}$. We solve the problem in terms of obstructions and a certain algebraic diagram of groups (see Remarks 1.1 and Proposition 1.4). The fundamental groups of several spaces which are relevant to our algebraic diagram are computed (see Propositions 1.8 and 1.9). In Section 2 we restrict to the case where the fiber is a torus. We classify all $T$-bundles over $S^{1}$ and bundle maps which, when restricted to the fiber, can be deformed to a fixed point free map. This is Theorem 2.1. Then we compute the fundamental group of $T$-bundles minus the zero section $S^{1}$. In terms of generators of this group, we obtain a system of equations which has a solution if and only if the map can be deformed fiberwise to a fixed point free map. This is Theorem 2.2. In Section 3 we discuss what we call the main equation, i.e. an equation such that the existence of its solution is equivalent to the fact that the corresponding fiber-preserving map can be deformed to a fixed point free map. First we reduce the study of all those equations to the study a certain family of equations. This is done in Theorem 3.3 and Corollary 3.4. The main equation can be regarded as an equation in a subgroup of the free group on two generators. Then we consider this equation in the abelianization of this group and deduce some necessary conditions for the existence of a solution. This is Corollary 3.8. In Section 4 we prove the main result, which is Theorem 4.1. The calculation is done for all six cases according to the classification of fiber-preserving maps.

\section{PRELIMINARY AND GENERAL RESULTS}

1.1. The general problem. Let $f: E \rightarrow E$ be a fiber-preserving map over $B$. When is $f$ deformable over $B$ to a fixed point free map $g$ by a fiberwise homotopy over $B$ ? 
E. Fadell and S. Husseini [F-H-81] considered this problem in the case where the fiber $F$, the base space $B$ and the total space $M$ are closed manifolds. They considered the fiber square $M \times_{B} M \rightarrow M$, i.e. the pull back fiber bundle of $p: M \rightarrow B$ by $p: M \rightarrow B$. Then the inclusion $M \times_{B} M-\Delta \rightarrow M \times_{B} M$, where $\Delta$ is the diagonal in $M \times_{B} M$, is replaced by the fiber bundle $q: E_{B}(M) \rightarrow M \times{ }_{B} M$, whose fiber is denoted by $\mathcal{F}$. So we have the following diagram:

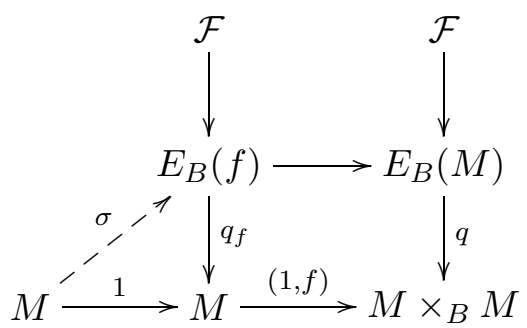

where $q_{f}: E_{B}(f) \rightarrow M$ is the fiber bundle induced from $q$ by $(1, f)$. From [F-H-81] we have

THEOREM 1.1. The map $f$ is deformable to a fixed point free map $g$ over $B$ if and only if there exists a lift $\sigma$ in diagram (1.1).

Remarks 1.1. (1) The fiber $\mathcal{F}$ has homotopy groups

$$
\pi_{j-1}(\mathcal{F}) \cong \pi_{j}\left(M \times{ }_{B} M, M \times{ }_{B} M-\Delta\right) \cong \pi_{j}(F, F-x),
$$

where $x$ is a point in $F$.

(2) Let $\operatorname{dim} F=k$. If $k>2$, in [F-H-81] classical obstruction theory was used to find a cross section. Hence, there is the primary obstruction to finding a cross section:

$$
\mathcal{O}_{B}(f) \in H^{k}\left(M ;\left\{\pi_{k-1}(\mathcal{F})\right\}\right),
$$

which is the primary (obstruction) fixed point index of $f$. This class is the obstruction to constructing an extension of a cross section from the $(k-1)$-skeleton to the $k$-skeleton of $M$. There may be other obstructions that preclude finding a global cross section.

(3) When the dimension of the fiber is two, in [Pe-97] a cohomology class $\mathcal{A}_{B}(f) \in H^{2}\left(M ;\left\{H_{1}(\mathcal{F})\right\}\right)$ is defined, called the abelianized obstruction to deforming $f$ over $B$ to a fixed point free map, where $\left\{H_{1}(\mathcal{F})\right\}$ is the induced abelianized local system of coefficients on $M$.

(4) When the fiber is the sphere $S^{2}$, then $\pi_{2}\left(S^{2}, S^{2}-x\right)$ is isomorphic to $\mathbb{Z}$ and we have a primary obstruction defined in dimension 2 . So this case can be treated as in item (2). 
(5) When the fiber is a surface with Euler characteristic $\leq 0$ then we will see by Proposition 1.5 that it is enough to build a cross section over the 2-skeleton. Proposition 1.6 gives a necessary and sufficient condition for the existence of such a partial section.

1.2. Classification of surface bundles over $S^{1}$. Let $S$ be a closed surface and $\phi: S \rightarrow S$ a homeomorphism which has one fixed point denoted by $x_{0}$ (in Corollary 1.3, we will see that there is no loss of generality in making such a hypothesis). We denote by $M(\phi)$ the quotient space obtained from $S \times I$ by identifying $(x, 0)$ with $(\phi(x), 1)$. The elements of $M(\phi)$ are denoted by $\langle x, t\rangle$. This space is an $S$-bundle over $S^{1}$ with fiber $S$ and projection $p: M(\phi) \rightarrow S^{1}=I / 0 \sim 1$, given by $p(\langle x, t\rangle)=\langle t\rangle$.

Proposition 1.2. Let $\phi_{1}, \phi_{2}: S \rightarrow S$ be two homeomorphisms. Then $M\left(\phi_{1}\right)$ is homeomorphic to $M\left(\phi_{2}\right)$ by a fiber-preserving homeomorphism over $S^{1}$ if and only if $\phi_{1}$ is isotopic to a conjugate of $\phi_{2}$.

Proof. Suppose that $\phi_{1}$ and $h \circ \phi_{2} \circ h^{-1}$ are isotopic. So we have a map $G: S \times I \rightarrow S \times I$ such that $G(, 0)=\phi_{1}$ and $G(, 1)=h \circ \phi_{2} \circ h^{-1}$. Let $G^{\prime}(, t)=h^{-1} \circ G(, t), t \in\{0,1\}$. We have

$$
G^{\prime}(, 1) \circ \phi_{1}=h^{-1} \circ h \circ \phi_{2} \circ h^{-1} \circ \phi_{1}=\phi_{2} \circ h^{-1} \circ \phi_{1}=\phi_{2} \circ G^{\prime}(, 0) .
$$

Hence we have a homeomorphism over $S^{1}$ between $M\left(\phi_{1}\right)$ and $M\left(\phi_{2}\right)$. The converse is similar and we leave its proof to the reader.

Corollary 1.3. The classes of $S$-bundles over $S^{1}$ are classified by the conjugacy classes of isotopy classes of homeomorphisms which preserve base points.

Proof. From [F-L-P-79, exposé 2] we know that every homeomorphism is isotopic to a homeomorphism which is base-point-preserving. So the result follows from Proposition 1.2.

1.3. Fixed point free fiber maps of surface bundles over $S^{1}$. We will assume from now on that the surface has Euler characteristic $\leq 0$. If $f: M(\phi) \rightarrow M(\phi)$ is a map over $S^{1}$, we define $f_{0}: S \rightarrow S$ by $f_{0}(x)=y$ if $f(\langle x, 0\rangle)=\langle y, 0\rangle$. This map is well defined since $\left\langle y_{1}, 0\right\rangle=\left\langle y_{2}, 0\right\rangle$ if and only if $y_{1}=y_{2}$.

Proposition 1.4. The map $\phi \circ f_{0} \circ \phi^{-1}$ is homotopic to $f_{0}$. Conversely, if $f_{0}: S \rightarrow S$ is a map with $\phi \circ f_{0} \circ \phi^{-1}$ homotopic to $f_{0}$, then there exists a map $f: M(\phi) \rightarrow M(\phi)$ over $S^{1}$ such that $f$ restricted to the fiber is $f_{0}$.

Proof. Define $f_{1}(x)=y$ if $f(\langle x, 1\rangle)=\langle y, 1\rangle$. Since $\langle x, 0\rangle=\langle\phi(x), 1\rangle$, it follows that $\left\langle f_{0}(x), 0\right\rangle=\left\langle f_{1} \circ \phi(x), 1\right\rangle$, which implies that $f_{1}=\phi \circ f_{0} \circ \phi^{-1}$. Now we observe that if $t \notin\{0,1\}$ then $f(\langle x, t\rangle)=\langle g(x, t), t\rangle$. Extending 
$g$ to a map $\bar{g}: S \times I \rightarrow S$ by continuity, we have $\bar{g}(x, 0)=f_{0}(x)$ and $\bar{g}(x, 1)=f_{1}(x)$, and the first part follows. The converse is quite similar. Define $f$ by the homotopy which connects $f_{0}$ and $\phi \circ f_{0} \circ \phi^{-1}$.

Proposition 1.5. If $M=M(\phi)$, then there exists a cross section $\sigma$ (see diagram (1.1)) over $M$ if and only if it exists over the 2-skeleton.

Proof. One direction is clear. So suppose that we have a cross section over the 2 -skeleton. Since the homotopy groups of the pair $(S, S-y)$ vanish in dimensions greater than or equal to 3 , by a standard obstruction argument the result follows.

Proposition 1.6. There is a cross section over the 2-skeleton if and only if the following diagram of fundamental groups admits a lifting $\psi$ :

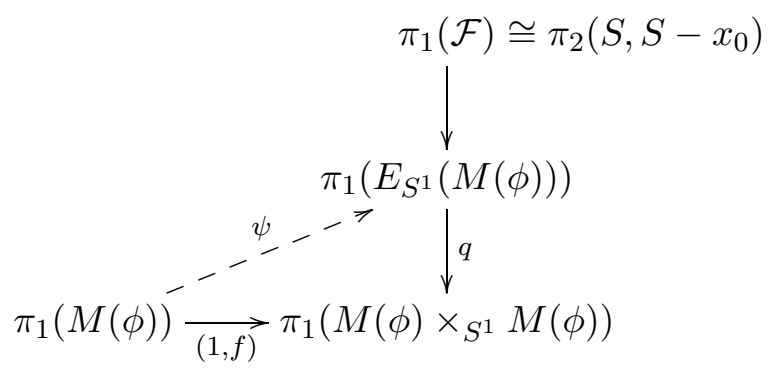

Proof. This follows from [Ba-77, Theorem 4.3.1, p. 265].

Let $s_{0}: S^{1} \rightarrow M(\phi)$ be given by $s_{0}(t)=\left\langle x_{0}, t\right\rangle$. This is a section of the bundle $p: M(\phi) \rightarrow S^{1}$, hence $1 \times\left(s_{0} \circ p\right)$ is also a section for the pullback $M(\phi) \times{ }_{S^{1}} M(\phi) \rightarrow M(\phi)$. With respect to these sections we have:

Proposition 1.7. We have a short exact sequence $1 \rightarrow \pi_{1}(S) \rightarrow$ $\pi_{1}(M(\phi)) \rightarrow \pi_{1}\left(S^{1}\right) \rightarrow 1$ which splits, and the action $\mathbb{Z} \rightarrow \operatorname{Aut}\left(\pi_{1}(S)\right)$ which comes from the section $s_{0}$ is given by $c \cdot \alpha=c \alpha c^{-1}=\phi_{\#}(\alpha)$, where $c=p_{\#}\left\langle s_{0}\right\rangle$ is the generator of $\pi_{1}\left(S^{1}\right)$. Hence $\pi_{1}(M(\phi)) \cong \pi_{1}(S) \rtimes \mathbb{Z}$, a semidirect product.

Proof. By the homotopy long exact sequence of the fibration, the short exact sequence follows since the base and the fiber are $K(\pi, 1)$ 's. Since $\mathbb{Z}$ is free the short exact sequence splits. Hence it remains to calculate the action. Let $\gamma: I \rightarrow S \times I$ be the path $\gamma(t)=\left(x_{0}, t\right)$. The loop obtained by the juxtaposed paths $\gamma *(\phi(\alpha), 1) * \gamma^{-1}$ is homotopic to the loop $(\phi(\alpha), 0)$. In the quotient space $M(\phi)$, this leads to $c \cdot \alpha=c \alpha c^{-1}=\phi_{\#}(\alpha)$, and the result follows.

Proposition 1.8. The fundamental group $\pi_{1}\left(M(\phi) \times{ }_{S^{1}} M(\phi)\right)$ is isomorphic to the semidirect product $\pi_{1}(S) \rtimes \pi_{1}(M(\phi))$. Further, the action of $\pi_{1}(M(\phi))$ on $\pi_{1}(S)$ is given by $\beta \cdot \alpha=\beta \alpha \beta^{-1}=p_{\#}(\beta) \cdot \alpha$. The last action 
is the one which comes from the bundle $p: M(\phi) \rightarrow S^{1}$, i.e. the action is given by the following composition:

$$
\pi_{1}(M(\phi)) \stackrel{p_{\#}}{\longrightarrow} \pi_{1}\left(S^{1}\right) \stackrel{\Gamma}{\rightarrow} \operatorname{Aut}\left(\pi_{1}(S)\right)
$$

where if we denote by $c$ the generator of $\pi_{1}\left(S^{1}\right)$ then $\Gamma(c)=\phi$, so that if $p_{\#}(\beta)=c^{k}$ then $p_{\#}(\beta) \circ \alpha=\phi^{k}(\alpha)$.

Proof. The result follows by naturality and the fact that $M(\phi) \times_{S^{1}} M(\phi)$ $\rightarrow M(\phi)$ is the pullback of $p: M(\phi) \rightarrow S^{1}$. A splitting of the short exact sequence

$$
0 \rightarrow \pi_{1}(S) \stackrel{i_{1 \#}}{\longrightarrow} \pi_{1}\left(M(\phi) \times_{S^{1}} M(\phi)\right) \stackrel{p_{2 \#}}{\longrightarrow} \pi_{1}(M(\phi)) \rightarrow 0
$$

is $S_{1}=\left(s_{0} \circ p, 1_{M(\phi)}\right) \#$ and the isomorphism $\Phi_{1}: \pi_{1}\left(M(\phi) \times_{S^{1}} M(\phi)\right) \rightarrow$ $\pi_{1}(S) \rtimes \pi_{1}(M(\phi))$ is given by $\Phi_{1}(\gamma)=\left(i_{1 \#}^{-1}\left(\gamma \cdot S_{1} \circ p_{2 \#}\left(\gamma^{-1}\right)\right), p_{2 \#}(\gamma)\right)$.

Let $\sigma\left(s_{0}\right)$ be the subset of $M(\phi)$ given by $\left\langle x_{0}, t\right\rangle, 0 \leq t \leq 1$. So we have the bundles $p: M(\phi)-\sigma\left(s_{0}\right) \rightarrow S^{1}$, where we denote the projection also by $p$ and the fiber is $S-x_{0}$. Another useful space is the pullback $M(\phi) \times_{S^{1}}\left(M(\phi)-\sigma\left(s_{0}\right)\right)$, and we will calculate its fundamental group.

Proposition 1.9. The fundamental group $\pi_{1}\left(M(\phi) \times_{S^{1}}\left(M(\phi)-\sigma\left(s_{0}\right)\right)\right)$ is isomorphic to the semidirect product $\pi_{1}(S) \rtimes \pi_{1}\left(M(\phi)-\sigma\left(s_{0}\right)\right)$. Further, the action of $\pi_{1}\left(M(\phi)-\sigma\left(s_{0}\right)\right)$ on $\pi_{1}(S)$ is given by $\beta \cdot \alpha=\beta \alpha \beta^{-1}=$ $p_{\#}(\beta) \cdot \alpha$, where the last action is the one which comes from the bundle $p: M(\phi)-\sigma\left(s_{0}\right) \rightarrow S^{1}$ as in Proposition 1.8.

Proof. Similar to the proof of Proposition 1.8. In this situation the fibration provides the short exact sequence

$$
0 \rightarrow \pi_{1}(S) \stackrel{i_{1 \#}}{\longrightarrow} \pi_{1}\left(M(\phi) \times_{S^{1}}\left(M(\phi)-\sigma\left(s_{0}\right)\right)\right) \stackrel{p_{2 \#}}{\longrightarrow} \pi_{1}\left(M(\phi)-\sigma\left(s_{0}\right)\right) \rightarrow 0
$$

and the homomorphism $s_{2}=\left(s_{0} \circ p, 1_{M(\phi)-\sigma\left(s_{0}\right)}\right)_{\#}$ is a section, and we define an isomorphism

$$
\Phi_{2}: \pi_{1}\left(M(\phi) \times_{S^{1}}\left(M(\phi)-\sigma\left(s_{0}\right)\right)\right) \rightarrow \pi_{1}(S) \rtimes \pi_{1}\left(M(\phi)-\sigma\left(s_{0}\right)\right)
$$

by $\Phi_{2}(\gamma)=\left(i_{1 \#}^{-1}\left(\gamma \cdot S_{2} \circ p_{2 \#}\left(\gamma^{-1}\right)\right), p_{2 \#}(\gamma)\right)$. The result about the action follows by naturality.

The above proposition shows the relevance of $\pi_{1}\left(M(\phi)-\sigma\left(s_{0}\right)\right)$.

\section{THE TORUS CASE AND REDUCTIONS OF THE LIFTING}

In this section we restrict to the case where the fiber is the torus denoted by $T$. Features of the torus are used to facilitate computations. We use some homeomorphisms of the torus to describe all $T$-bundles over $S^{1}$. We also use the group structure of $T$ to simplify the analysis of our algebraic problem. 
Let $T$ be defined as the quotient space $\mathbb{R} \times \mathbb{R} / \mathbb{Z} \times \mathbb{Z}$; we denote by $\left(\begin{array}{l}x \\ y\end{array}\right)$ and $\left[\begin{array}{l}x \\ y\end{array}\right]$ the elements of $\mathbb{R} \times \mathbb{R}$ and $T$, respectively.

Let $A$ be a homeomorphism of $T$ induced by an operator in $\mathbb{R}^{2}$ that preserves $\mathbb{Z} \times \mathbb{Z}$. We identify $A$ with a matrix with integer coefficients and determinant either 1 or -1 .

As in Section 1 let $M A$ be the quotient space of $T \times[0,1]$, where we identify $\left(\left[\begin{array}{l}x \\ y\end{array}\right], 0\right)$ with $\left(\left[A\left(\begin{array}{l}x \\ y\end{array}\right)\right], 1\right)$.

The class of $\left(\left[\begin{array}{l}x \\ y\end{array}\right], t\right)$ in the quotient is denoted by $\left\langle\left[\begin{array}{l}x \\ y\end{array}\right], t\right\rangle$. The space $M A$ is a fiber bundle over the circle $S^{1}$, where the fiber is the torus. The projection map $p: M A \rightarrow S^{1}$ is given by $p\left(\left\langle\left[\begin{array}{l}x \\ y\end{array}\right], t\right\rangle\right)=\langle t\rangle \in[0,1] /_{0 \simeq 1} \cong S^{1}$.

Let $f: M A \rightarrow M A$ be a map over $S^{1}$, i.e. $p \circ f=p$, and we consider the question raised in 1.1: when is $f$ deformable to a fixed point free map $g$ by a fiberwise homotopy over $S^{1}$ ?

From Section 1 we know that the problem is equivalent to finding a lift in the following algebraic diagram:

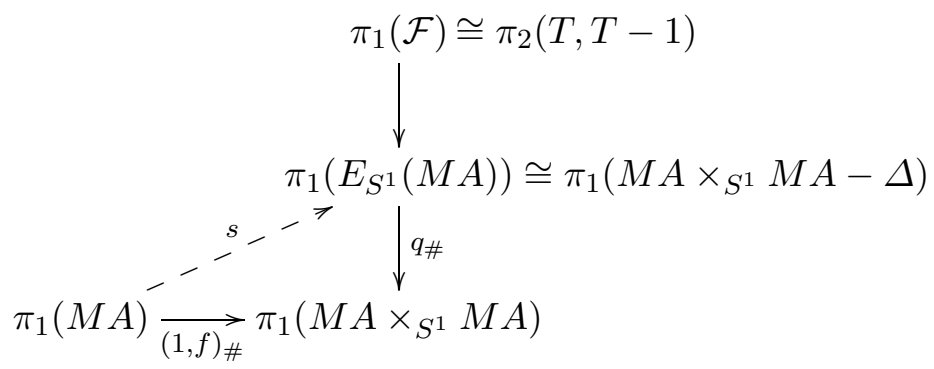

with suitable base points.

The base point of the domain of $f$ is $\left\langle\left[\begin{array}{l}0 \\ 0\end{array}\right], 0\right\rangle$, denoted by $\mathbf{0}$, and we can suppose that $f(\mathbf{0})$ is $\left\langle\left[\begin{array}{c}q \\ q\end{array}\right], 0\right\rangle$, denoted by $\mathbf{q}$. Otherwise we can replace the map $f$ by a map $f_{1}$ homotopic to $f$ which has the property above. This can be done using the homotopy lifting property of the fibration $p: M(A) \rightarrow S^{1}$.

We denote by $B$ the matrix of the homomorphism induced on the fundamental group by the restriction of $f$ to the fiber $T$. The next theorem provides a relationship between the matrices $A$ and $B$.

Theorem 2.1. (1) $\pi_{1}(M A, \mathbf{0})=\left\langle a, b, c_{0}:[a, b]=1, c_{0} a c_{0}{ }^{-1}=a^{a_{1}} b^{a_{2}}\right.$, $\left.c_{0} b c_{0}{ }^{-1}=a^{a_{3}} b^{a_{4}}\right\rangle$.

(2) B commutes with A.

(3) If $f$ restricted to the fiber is deformable to a fixed point free map then the determinant of $B-I$ is zero, where $I$ is the identity matrix.

(4) If $v$ is an eigenvector of $B$ associated to 1 (for $B \neq \mathrm{Id})$ then $A(v)$ is also an eigenvector of $B$ associated to 1.

(5) Consider $w=A(v)$ if the pair $v, w$ generates $\mathbb{Z} \times \mathbb{Z}$, otherwise let $w$ be another vector so that $v, w$ span $\mathbb{Z} \times \mathbb{Z}$. Define the linear operator 
$P: \mathbb{R} \times \mathbb{R} \rightarrow \mathbb{R} \times \mathbb{R}$ by $P(v)=\left(\begin{array}{l}1 \\ 0\end{array}\right)$ and $P(w)=\left(\begin{array}{l}0 \\ 1\end{array}\right)$. Consider an isomorphism of fiber bundles (also denoted by $P$ ) $P: M A \rightarrow M A^{1}$, where $A^{1}=P \circ A \circ P^{-1}$. Hence $M A$ is homeomorphic to $M A^{1}$ over $S^{1}$. Then we have one of the cases of the table below, with $B^{1}=P \circ B \circ P^{-1}$ and $B^{1} \neq I$, except in case $I$ :

\begin{tabular}{|c|c|c|c|}
\hline Case I & $\begin{array}{c}A^{1}=\left(\begin{array}{cc}a_{1} & a_{3} \\
a_{2} & a_{4}\end{array}\right), \quad B^{1}=\left(\begin{array}{ll}1 & 0 \\
0 & 1\end{array}\right) \\
a_{3} \neq 0\end{array}$ & Case IV & $\begin{array}{c}A^{1}=\left(\begin{array}{cc}1 & a_{3} \\
0 & -1\end{array}\right), \quad B^{1}=\left(\begin{array}{ll}1 & b_{3} \\
0 & b_{4}\end{array}\right) \\
a_{3}\left(b_{4}-1\right)=-2 b_{3}\end{array}$ \\
\hline Case II & $A^{1}=\left(\begin{array}{ll}1 & 0 \\
0 & 1\end{array}\right), \quad B^{1}=\left(\begin{array}{ll}1 & b_{3} \\
0 & b_{4}\end{array}\right)$ & Case V & $\begin{array}{c}A^{1}=\left(\begin{array}{cc}-1 & a_{3} \\
0 & -1\end{array}\right), \quad B^{1}=\left(\begin{array}{ll}1 & b_{3} \\
0 & b_{4}\end{array}\right) \\
a_{3}\left(b_{4}-1\right)=0\end{array}$ \\
\hline Case III & $A^{1}=\left(\begin{array}{cc}1 & a_{3} \\
0 & 1\end{array}\right), \quad B^{1}=\left(\begin{array}{cc}1 & b_{3} \\
0 & 1\end{array}\right)$ & Case VI & $\begin{array}{c}A^{1}=\left(\begin{array}{cc}-1 & a_{3} \\
0 & 1\end{array}\right), \quad B^{1}=\left(\begin{array}{ll}1 & b_{3} \\
0 & b_{4}\end{array}\right) \\
a_{3}\left(b_{4}-1\right)=2 b_{3}\end{array}$ \\
\hline
\end{tabular}

Proof. Consider the following loops in $M A$ with base point $\mathbf{0}$ :

$$
a(t)=\left\langle\left[\begin{array}{l}
t \\
0
\end{array}\right], 0\right\rangle, \quad b(t)=\left\langle\left[\begin{array}{l}
0 \\
t
\end{array}\right], 0\right\rangle, \quad c_{0}(t)=\left\langle\left[\begin{array}{l}
0 \\
0
\end{array}\right], t\right\rangle,
$$

for $t \in[0,1]$. From Proposition 1.7 it follows that $\pi_{1}(M A) \cong \pi_{1}(T) \rtimes \mathbb{Z}$, and a presentation is $\pi_{1}(M A, \mathbf{0})=\left\langle a, b, c_{0}:[a, b]=1, c_{0} a c_{0}{ }^{-1}=a^{a_{1}} b^{a_{2}}, c_{0} b c_{0}{ }^{-1}=\right.$ $\left.a^{a_{3}} b^{a_{4}}\right\rangle$.

Recall that $B=\left(\begin{array}{ll}b_{1} & b_{3} \\ b_{2} & b_{4}\end{array}\right)$ is the matrix of the homomorphism induced on the fundamental group of the fiber $T$ by the restriction of $f$ to $T$, and $f$ is a map over $S^{1}$. Then the induced homomorphism $f_{\#}$ on $\pi_{1}(M A, \mathbf{0})$ is given by $f_{\#}(a)=a^{b_{1}} b^{b_{2}}, f_{\#}(b)=a^{b_{3}} b^{b_{4}}$ and $f_{\#}(c)=a^{c_{1}} b^{c_{2}} c$.

Since $f$ is a map over $S^{1}$ it follows from Proposition 1.4 that $B$ commutes with $A$.

Since $f$ is deformable over $S^{1}$ to a fixed point free map $g$, the Lefschetz number of $\left.f\right|_{T}$ is 0 , so $\operatorname{det}(B-I)=0$. Now (4) follows from (2). To prove $(5)$ define $P\left(\left\langle\left[\begin{array}{l}x \\ y\end{array}\right], t\right\rangle\right)=\left\langle\left[P\left(\begin{array}{l}x \\ y\end{array}\right)\right], t\right\rangle$. Thus $P_{\#} f_{\#} P_{\#}^{-1}: \pi_{1}\left(M A^{1}, \mathbf{0}\right) \rightarrow$ $\pi_{1}\left(M A^{1}, \mathbf{0}\right)$ maps the generator $a(t)$ to $a(t)$. So the first column of $B^{1}$ is $\left[\begin{array}{l}1 \\ 0\end{array}\right]$ and therefore $B^{1}=\left(\begin{array}{ll}1 & b_{3} \\ 0 & b_{4}\end{array}\right)$.

Now the commutativity of $B^{1}$ with $A^{1}$ yields the table. We remark that Case I occurs when $v$ and $A(v)$ span $\mathbb{Z} \times \mathbb{Z}$.

2.1. The fiber bundle isomorphism $h$. Now, we define a fiber bundle isomorphism $h: M A \times_{S^{1}} M A \rightarrow M A \times_{S^{1}} M A$ by

$$
h\left(\left\langle\left[\begin{array}{l}
x \\
y
\end{array}\right], t\right\rangle,\left\langle\left[\begin{array}{l}
x^{\prime} \\
y^{\prime}
\end{array}\right], t\right\rangle\right)=\left(\left\langle\left[\begin{array}{l}
x \\
y
\end{array}\right], t\right\rangle,\left\langle\left[\begin{array}{l}
x^{\prime} \\
y^{\prime}
\end{array}\right]-\left[\begin{array}{l}
x \\
y
\end{array}\right], t\right\rangle\right) .
$$

This isomorphism maps $M A \times_{S^{1}} M A-\Delta$ onto $M A \times_{S^{1}}\left(M A-S^{1}\right)$. This last space is the fiber square of the maps $\left.p\right|_{M A-S^{1}}: M A-S^{1} \rightarrow S^{1}$ and $p: M A \rightarrow S^{1}$, and the circle $S^{1}$ in $M A$ is the image of $c_{0}(t)=\left\langle\left[\begin{array}{l}0 \\ 0\end{array}\right], t\right\rangle$ for all $t \in[0,1]$. 
So, we have the commutative diagram

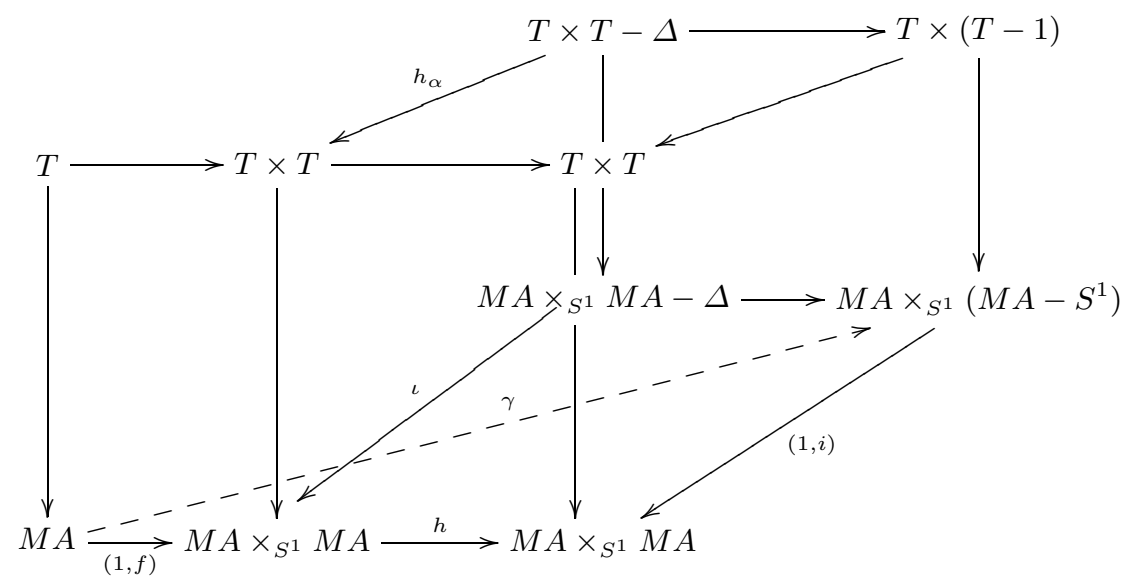

Therefore, the problem of existence of the section $\sigma$ in diagram (1.1) is equivalent to the existence of a lift $\gamma$. By the same argument as in Proposition 1.6, the existence of $\gamma$ is equivalent to the existence of a certain homomorphism on the level of fundamental groups. Since $M A \times_{S^{1}}\left(M A-S^{1}\right)$ is the total space of the pull back of $p$ by $\left.p\right|_{M A-S^{1}}$, by the universal property of the pullback and using the isomorphisms $\Phi_{1}$ and $\Phi_{2}$ of Propositions 1.8 and 1.9 respectively, it is easy to show the equivalence of the existence of the lifting homomorphism in diagram (2.1) and the lifting homomorphism $\Gamma$ that makes the diagram below commutative:

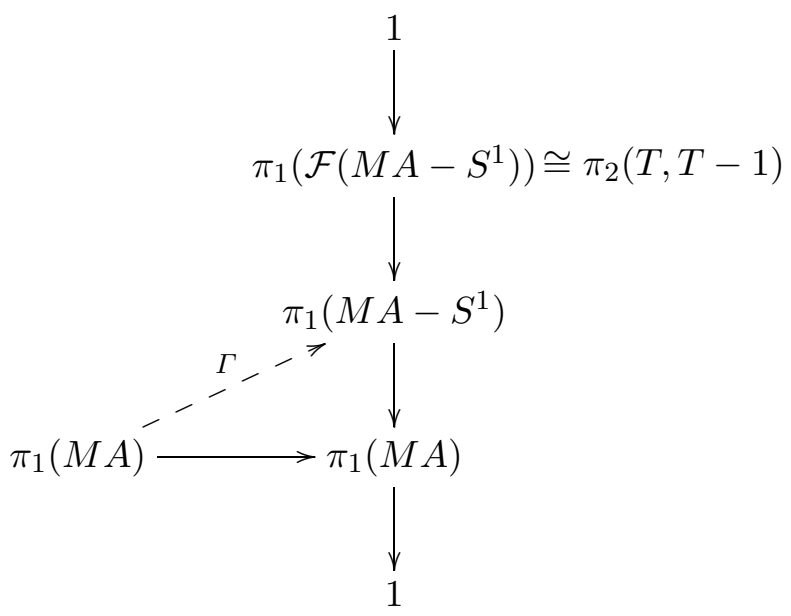

We will describe the horizontal map $\left(p_{2} \circ h \circ(1, f)\right)_{\#}: \pi_{1}(M A) \rightarrow \pi_{1}(M A)$ in the next theorem in terms of generators. Here $p_{2}: M A \times_{S^{1}} M A \rightarrow M A$ denotes the projection on the second factor. 
2.2. The generators of the fundamental groups in diagram (2.2). The next theorem describes the groups and the maps of diagram (2.2). Consider the following loops in $M A$ with base point $\mathbf{0}$, and loops in $M A-S^{1}$ or $M A$ with base point $\mathbf{q}$ with $q$ small positive and $\left[\begin{array}{l}0 \\ 0\end{array}\right] \neq\left[\begin{array}{l}q \\ q\end{array}\right]$ :

$$
\begin{aligned}
& a(t)=\left\langle\left[\begin{array}{l}
t \\
0
\end{array}\right], 0\right\rangle, \quad b(t)=\left\langle\left[\begin{array}{l}
0 \\
t
\end{array}\right], 0\right\rangle, \quad c_{0}(t)=\left\langle\left[\begin{array}{l}
0 \\
0
\end{array}\right], t\right\rangle, \\
& e(t)=\left\langle\left[\begin{array}{c}
q+t \\
q
\end{array}\right], 0\right\rangle, \quad d(t)=\left\langle\left[\begin{array}{c}
q \\
q+t
\end{array}\right], 0\right\rangle, \quad c(t)=\langle[\gamma(t)], t\rangle,
\end{aligned}
$$

where $\gamma(t)$ is $(1-t)\left(\begin{array}{l}q \\ q\end{array}\right)+t\left(A\left(\begin{array}{l}q \\ q\end{array}\right)\right)$ if $A\left(\begin{array}{c}q \\ q\end{array}\right) \neq\left(\begin{array}{c}-q \\ -q\end{array}\right)$; otherwise, it is an arc which runs counterclockwise around the origin from $\left(\begin{array}{c}q \\ q\end{array}\right)$ to $\left(\begin{array}{c}-q \\ -q\end{array}\right)$. Here, we suppose that $\left(A\left(\begin{array}{l}q \\ q\end{array}\right)\right)$ belongs to the square centered at the origin $(0,0)$ with side two.

Finally, let $W(t)$ be the circle around the origin having $(q, q)$ as the base point and oriented counterclockwise.

We denote the homotopy classes of the loops $a(t), b(t), c_{0}(t)$ in $\pi_{1}(M A, \mathbf{0})$ by $a, b, c_{0}$ respectively. We denote the homotopy classes of the loops $e(t), d(t)$, $c(t)$ in $\pi_{1}\left(M A-S^{1}, \mathbf{q}\right)$ by $e, d, c$ respectively, and in $\pi_{1}(M A, \mathbf{q})$ by $\bar{e}, \bar{d}, \bar{c}$ respectively.

The next theorem describes the homomorphisms of diagram (2.2).

Theorem 2.2. Let $A$ and $B$ be as in one of the six cases given by Theorem 2.1 and let $f_{\#}$ be the homomorphism induced by $f$ on the fundamental group. Then:

(1) $\pi_{1}\left(M A \times_{S^{1}} M A,(\mathbf{0}, \mathbf{q})\right)=\langle a, b, \bar{e}, \bar{d}, \hat{c}:[a, b]=1,[a, \bar{e}]=1,[a, \bar{d}]=1$, $[b, \bar{e}]=1,[b, \bar{d}]=1,[\bar{e}, \bar{d}]=1, \hat{c} a \hat{c}^{-1}=a^{a_{1}} b^{a_{2}}, \hat{c} b \hat{c}^{-1}=a^{a_{3}} b^{a_{4}}$, $\left.\hat{c} \bar{e} \hat{c}^{-1}=\bar{e}^{a_{1}} \bar{d}^{a_{2}}, \hat{c} \bar{d} \hat{c}^{-1}=\bar{e}^{a_{1}} \bar{d}^{a_{2}}\right\rangle$, where $\hat{c}$ is the homotopy class of

\begin{tabular}{|c|c|}
\hline & $\pi_{1}\left(M A-S^{1}, \mathbf{q}\right)$ \\
\hline Case II & $\begin{array}{c}A=\left(\begin{array}{ll}1 & 0 \\
0 & 1\end{array}\right), B=\left(\begin{array}{ll}1 & b_{3} \\
0 & b_{4}\end{array}\right) \text { with } b_{3} \neq 0 \text { or } b_{4} \neq 1 \\
\left\langle e, d, c: c e c^{-1}=e, c d c^{-1}=d\right\rangle\end{array}$ \\
\hline Case III & $\begin{array}{c}A=\left(\begin{array}{cc}1 & a_{3} \\
0 & 1\end{array}\right), B=\left(\begin{array}{cc}1 & b_{3} \\
0 & b_{4}\end{array}\right) \text { with } a_{3} \neq 0 \text { and } b_{3} \neq 0 \\
\left\langle e, d, c: c e c^{-1}=e, c d c^{-1}=e^{a_{3}} d\right\rangle\end{array}$ \\
\hline Case IV & $\begin{array}{c}A=\left(\begin{array}{ll}1 & a_{3} \\
0 & -1\end{array}\right), B=\left(\begin{array}{ll}1 & b_{3} \\
0 & b_{4}\end{array}\right) \text { with } a_{3}\left(b_{4}-1\right)=-2 b_{3} \text { and } b_{4} \neq 1 \\
\quad \text { if } a_{3} \geq-1 \text { then }\left\langle e, d, c: c e c^{-1}=e W, c d c^{-1}=d^{-1} e^{a_{3}}\right\rangle \\
\text { if } a_{3} \leq-2 \text { then }\left\langle e, d, c: c e c^{-1}=W e, c d c^{-1}=W d^{-1} e^{a_{3}} W^{-1}\right\rangle\end{array}$ \\
\hline Case V & $\begin{array}{c}A=\left(\begin{array}{cc}-1 & a_{3} \\
0 & -1\end{array}\right), B=\left(\begin{array}{ll}1 & b_{3} \\
0 & b_{4}\end{array}\right) \text { with } a_{3}\left(b_{4}-1\right)=0 \text { and } b_{3} \neq 0 \text { or } b_{4} \neq 1 \\
\quad \text { if } a_{3} \geq 1 \text { then }\left\langle e, d, c: c e c^{-1}=W^{-1} e^{-1}, c d c^{-1}=d^{-1} e^{a_{3}} W\right\rangle \\
\text { if } a_{3} \leq 0 \text { then }\left\langle e, d, c: c e c^{-1}=e^{-1} W^{-1}, c d c^{-1}=W d^{-1} e^{a_{3}}\right\rangle\end{array}$ \\
\hline Case VI & $\begin{array}{c}A=\left(\begin{array}{cc}-1 & a_{3} \\
0 & 1\end{array}\right), B=\left(\begin{array}{cc}1 & b_{3} \\
0 & b_{4}\end{array}\right) \text { with } a_{3}\left(b_{4}-1\right)=2 b_{3} \text { and } b_{4} \neq 1 \\
\left\langle e, d, c: c e c^{-1}=e^{-1}, c d c^{-1}=e^{a_{3}} d W^{-1}\right\rangle\end{array}$ \\
\hline
\end{tabular}
the loop given by the pair of loops $\left(c_{0}(t), \bar{c}(t)\right)$.

where $W$ is homotopic to the loop $e^{-1} d^{-1} e d$. 
(3) The homomorphism $\left(p_{2} \circ h \circ(1, f)\right)_{\#}$ is given by

$$
a \mapsto \bar{e}^{\left(b_{1}-1\right)} \bar{d}^{b_{2}}, \quad b \mapsto \bar{e}^{b_{3}} \bar{d}^{\left(b_{4}-1\right)}, \quad c_{0} \mapsto \bar{e}^{c_{1}} \bar{d}^{c_{2}} c .
$$

(4) The homomorphism $i_{\#}: \pi_{1}\left(M A-S^{1}\right) \rightarrow \pi_{1}(M A)$ is given by

$$
e \mapsto \bar{e}, \quad d \mapsto \bar{d}, \quad c \mapsto \bar{c} .
$$

(5) The homomorphism $\Gamma$ exists if and only if we can find elements $Z_{1}, Z_{2}, Z_{3} \in \operatorname{ker}\left(\pi_{1}(T-1) \rightarrow \pi_{1}(T)\right)$ such that

$$
\Gamma(a)=Z_{1} e^{b_{1}-1} d^{b_{2}}, \quad \Gamma(b)=Z_{2} e^{b_{3}} d^{b_{4}-1}, \quad \Gamma\left(c_{0}\right)=Z_{3} e^{c_{1}} d^{c_{2}} c,
$$

and

$$
\left\{\begin{array}{l}
{[\Gamma(a), \Gamma(b)]=1,} \\
\Gamma\left(c_{0}\right) \Gamma(a) \Gamma\left(c_{0}{ }^{-1}\right)=\Gamma\left(a^{a_{1}} b^{a_{2}}\right), \\
\Gamma\left(c_{0}\right) \Gamma(b) \Gamma\left(c_{0}{ }^{-1}\right)=\Gamma\left(a^{a_{3}} b^{a_{4}}\right) .
\end{array}\right.
$$

Proof. (1) It follows from Proposition 1.8 that

$$
\pi_{1}\left(M(A) \times{ }_{S^{1}} M(A),(\mathbf{0}, \mathbf{q})\right) \cong \pi_{1}(T, \mathbf{0}) \rtimes \pi_{1}(M(A), \mathbf{q})
$$

and a presentation is

$$
\begin{array}{r}
\langle a, b, \bar{e}, \bar{d}, \hat{c}:[a, b]=1,[a, \bar{e}]=1,[a, \bar{d}]=1, \\
{[b, \bar{e}]=1,[b, \bar{d}]=1,[\bar{e}, \bar{d}]=1,} \\
\hat{c} a \hat{c}^{-1}=a^{a_{1}} b^{a_{2}}, \hat{c} b \hat{c}^{-1}=a^{a_{3}} b^{a_{4}}, \\
\left.\hat{c} \bar{e} \hat{c}^{-1}=\bar{e}^{a_{1}} \bar{d}^{a_{2}}, \hat{c} \bar{d} \hat{c}^{-1}=\bar{e}^{a_{1}} \bar{d}^{a_{2}}\right\rangle .
\end{array}
$$

(2) We illustrate the cases V: $a_{3}=0$ and VI: $a_{3}>1$. For the other cases the calculation is similar and we leave the details to the reader. Recall that $W$ is the loop around the origin which runs counterclockwise. So it represents the class of the element $e^{-1} d^{-1} e d$.

CASE V: $a_{3}=0$. We are going to construct liftings of the paths $c, e$ and $c^{-1}$ to $\left(\mathbb{R}^{2}-(\mathbb{Z} \times \mathbb{Z})\right) \times I$, denoted by $\tilde{c}$, $\tilde{e}$ and $\tilde{c}^{-1}$, respectively, so that the projection of the juxtaposed path $\tilde{c} \tilde{e} \tilde{c}^{-1}$ on $M A-S^{1}$ is $c e c^{-1}$. In $\left(\mathbb{R}^{2}-(\mathbb{Z} \times \mathbb{Z})\right) \times I$ we give a homotopy of the path $\tilde{c} \tilde{e} \tilde{c}^{-1}$ to a new path which clearly projects to $e^{-1} W^{-1}$. Consider

$$
\begin{aligned}
\tilde{c}(t) & =(\gamma(t), t), \\
\tilde{e}(t) & =\left(A\left(\begin{array}{c}
q+t \\
q
\end{array}\right), 1\right), \\
\tilde{c}^{-1}(t) & =\left(\gamma(1-t)-\left(\begin{array}{l}
1 \\
0
\end{array}\right), 1-t\right) .
\end{aligned}
$$

The sequence of diagrams below shows a homotopy between $\tilde{c} \tilde{e} \tilde{c}^{-1}$ and a path in the face $\left(\mathbb{R}^{2}-(\mathbb{Z} \times \mathbb{Z})\right) \times 0$ of $\left(\mathbb{R}^{2}-(\mathbb{Z} \times \mathbb{Z})\right) \times I$. 

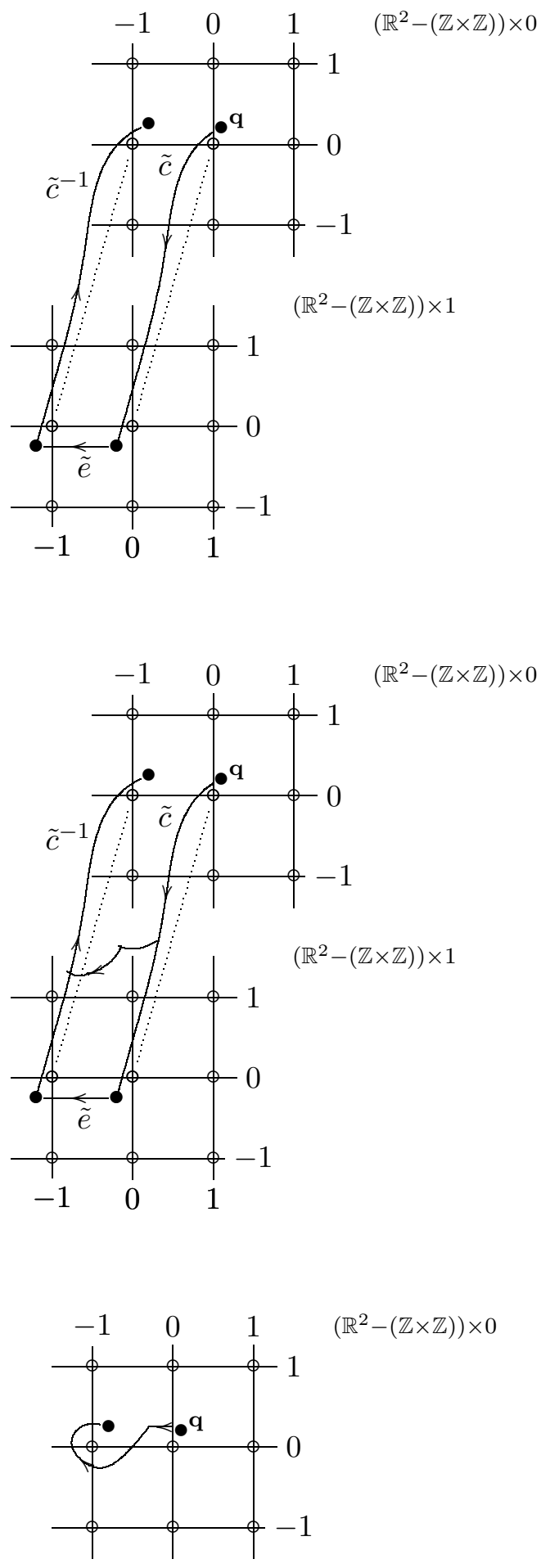

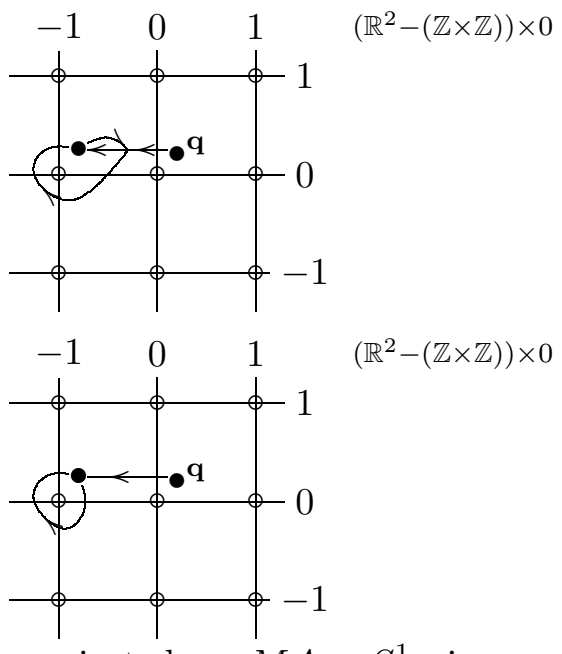

This last path when projected on $M A-S^{1}$ gives $e^{-1} W^{-1}$ and therefore $\operatorname{cec}^{-1}=e^{-1} W^{-1}$.

Now the relation $c d c^{-1}=W d^{-1}$ is obtained as follows. We are going to construct liftings of the paths $c, d$ and $c^{-1}$ to $\left(\mathbb{R}^{2}-(\mathbb{Z} \times \mathbb{Z})\right) \times I$, denoted by $\tilde{c}, \tilde{d}$ and $\tilde{c}^{-1}$, respectively, so that the projection of $\tilde{c} \tilde{d} \tilde{c}^{-1}$ on $M A-S^{1}$ is $c d c^{-1}$. In $\left(\mathbb{R}^{2}-(\mathbb{Z} \times \mathbb{Z})\right) \times I$ we give a homotopy of $\tilde{c} \tilde{d} \tilde{c}^{-1}$ to a new path which clearly projects to $W d^{-1}$.

Consider

$$
\begin{aligned}
\tilde{c}(t) & =(\gamma(t), t), \quad \tilde{d}(t)=\left(A\left(\begin{array}{c}
q \\
q+t
\end{array}\right), 1\right), \\
\tilde{c}^{-1}(t) & =\left(\gamma(1-t)-\left(\begin{array}{l}
0 \\
1
\end{array}\right), 1-t\right) .
\end{aligned}
$$

The sequence of diagrams below shows a homotopy between $\tilde{c} \tilde{d} \tilde{c}^{-1}$ and a path in the face $\left(\mathbb{R}^{2}-(\mathbb{Z} \times \mathbb{Z})\right) \times 0$ of $\left(\mathbb{R}^{2}-(\mathbb{Z} \times \mathbb{Z})\right) \times I$.

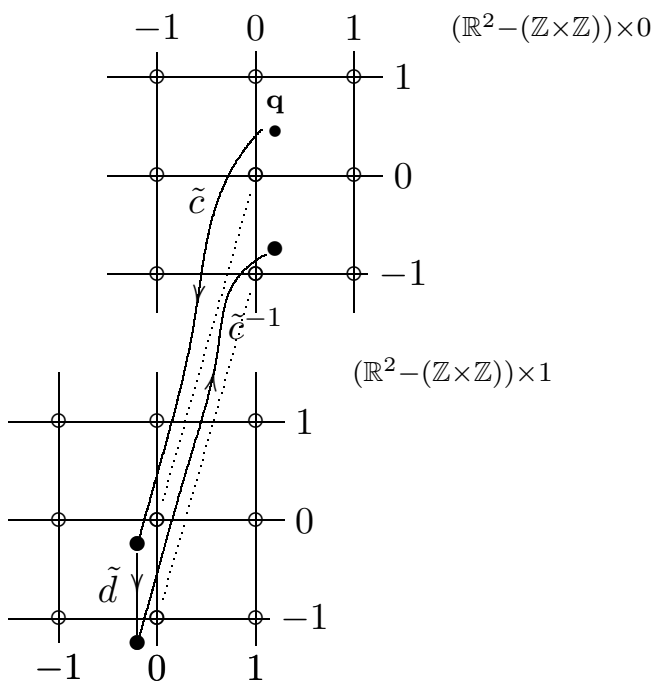




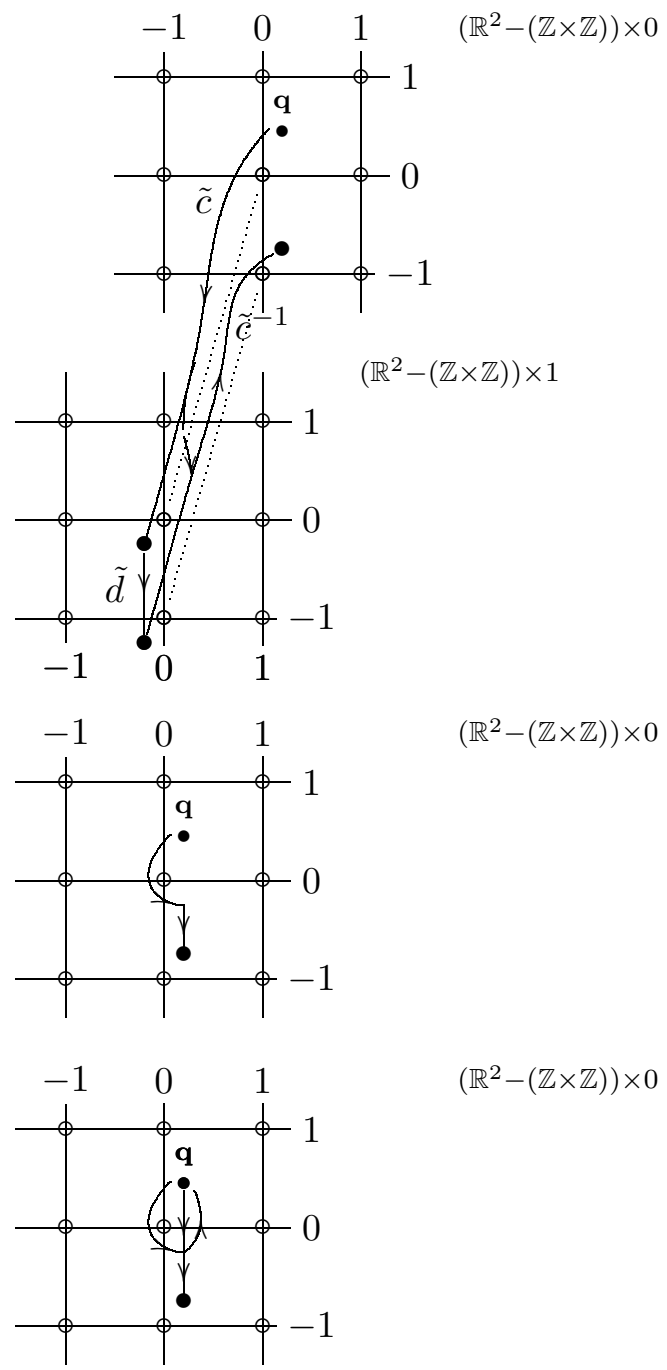

This last path when projected on $M A-S^{1}$ gives $W d^{-1}$ and therefore $c d c^{-1}=$ $W d^{-1}$.

CASE VI: $a_{3}>1$. We are going to construct liftings of the paths $c, e$ and $c^{-1}$ to $\left(\mathbb{R}^{2}-(\mathbb{Z} \times \mathbb{Z})\right) \times I$, denoted by $\tilde{c}, \tilde{e}$ and $\tilde{c}^{-1}$, respectively, so that the projection of $\tilde{c} \tilde{e} \tilde{c}^{-1}$ on $M A-S^{1}$ is $c e c^{-1}$. In $\left(\mathbb{R}^{2}-(\mathbb{Z} \times \mathbb{Z})\right) \times I$ we give a homotopy of $\tilde{c} \tilde{e} \tilde{c}^{-1}$ to a new path which clearly projects to $e^{-1}$.

Consider

$$
\tilde{c}(t)=(\gamma(t), t), \quad \tilde{e}(t)=\left(A\left(\begin{array}{c}
q+t \\
q
\end{array}\right), 1\right), \quad \tilde{c}^{-1}(t)=\left(\gamma(1-t)-\left(\begin{array}{l}
1 \\
0
\end{array}\right), 1-t\right) .
$$

The sequence of diagrams below shows a homotopy between $\tilde{c} \tilde{e} \tilde{c} \tilde{c}^{-1}$ and a path in the face $\left(\mathbb{R}^{2}-(\mathbb{Z} \times \mathbb{Z})\right) \times 0$ of $\left(\mathbb{R}^{2}-(\mathbb{Z} \times \mathbb{Z})\right) \times I$. 

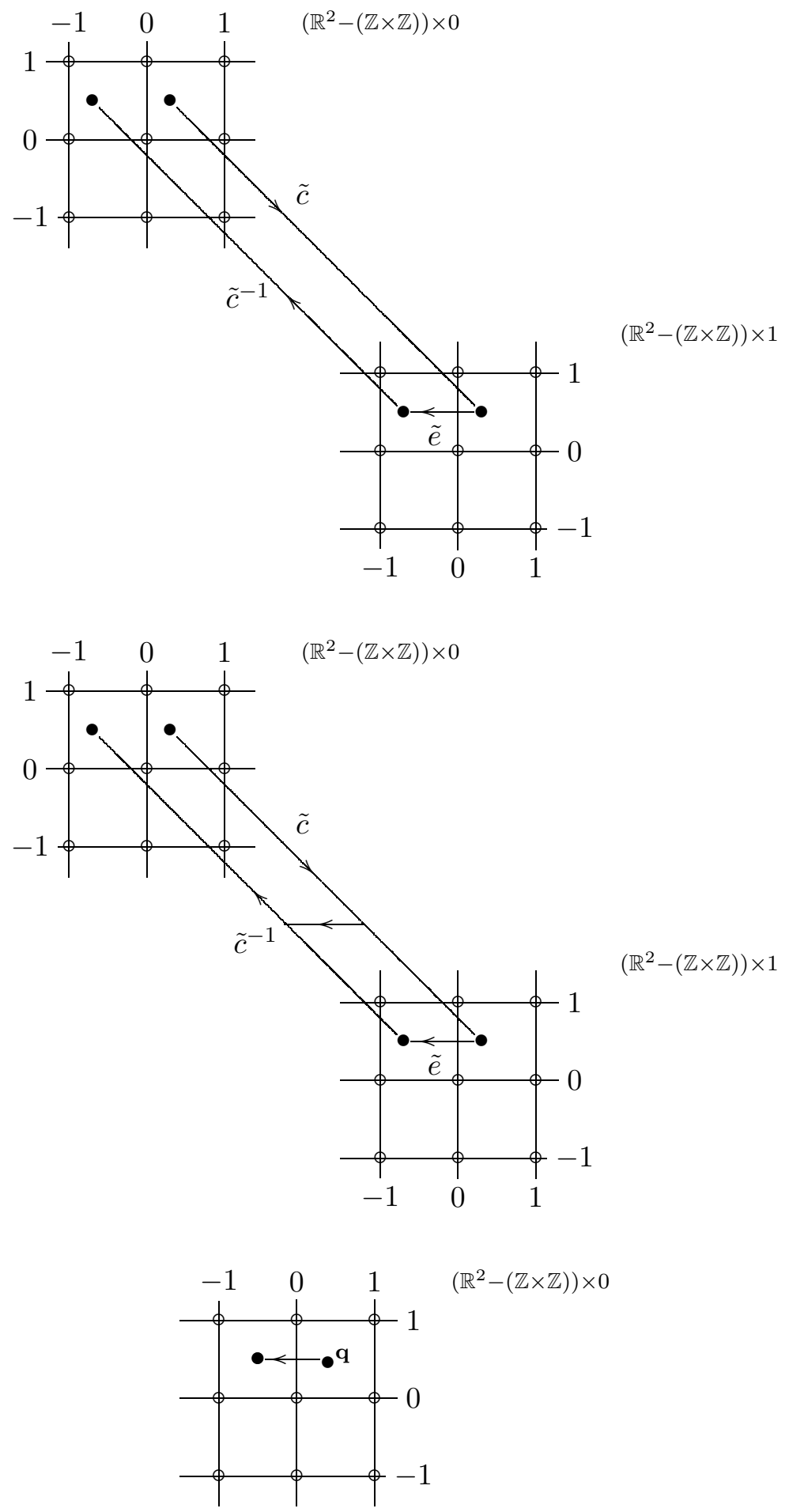
This last path when projected on $M A-S^{1}$ gives $e^{-1}$ and therefore $c e c^{-1}=$ $e^{-1}$.

Now the relation $c d c^{-1}=e^{a_{3}} d W^{-1}$ is obtained as follows. We are going to construct liftings of the paths $c, d$ and $c^{-1}$ to $\left(\mathbb{R}^{2}-(\mathbb{Z} \times \mathbb{Z})\right) \times I$, denoted by $\tilde{c}, \tilde{d}$ and $\tilde{c}^{-1}$, respectively, so that the projection of $\tilde{c} \tilde{d} \tilde{c}^{-1}$ on $M A-S^{1}$ is $c d c^{-1}$. In $\left(\mathbb{R}^{2}-(\mathbb{Z} \times \mathbb{Z})\right) \times I$ we give a homotopy of $\tilde{c} \tilde{e} \tilde{c}^{-1}$ to a new path which clearly projects to $e^{a_{3}} d W^{-1}$.

Consider

$$
\begin{aligned}
\tilde{c}(t) & =(\gamma(t), t), \\
\tilde{d}(t) & =\left(A\left(\begin{array}{c}
q \\
q+t
\end{array}\right), 1\right), \\
\tilde{c}^{-1}(t) & =\left(\gamma(1-t)+\left(\begin{array}{c}
a_{3} \\
1
\end{array}\right), 1-t\right) .
\end{aligned}
$$

We observe that since $\mathbf{q}$ was chosen such that $A_{\mathbf{q}}$ belongs to the square centered at the origin $(0,0)$ with side two, the path $\tilde{d}$ must pass by a point with $x$-coordinate between $a_{3}-1$ and $a_{3}$ and with $y$-coordinate 1 .

The sequence of diagrams belows shows a homotopy between $\tilde{c} \tilde{d} \tilde{c}^{-1}$ and a path in the face $\left(\mathbb{R}^{2}-(\mathbb{Z} \times \mathbb{Z})\right) \times 0$ of $\left(\mathbb{R}^{2}-(\mathbb{Z} \times \mathbb{Z})\right) \times I$.

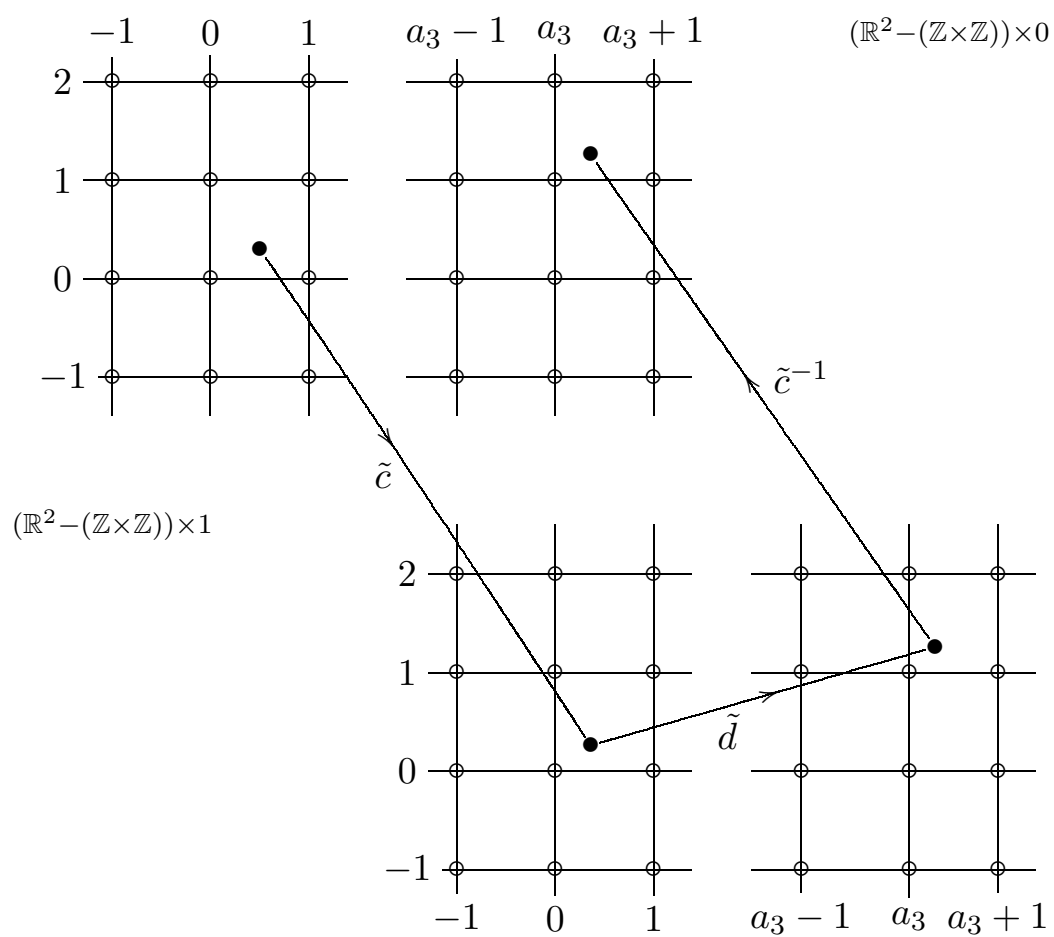



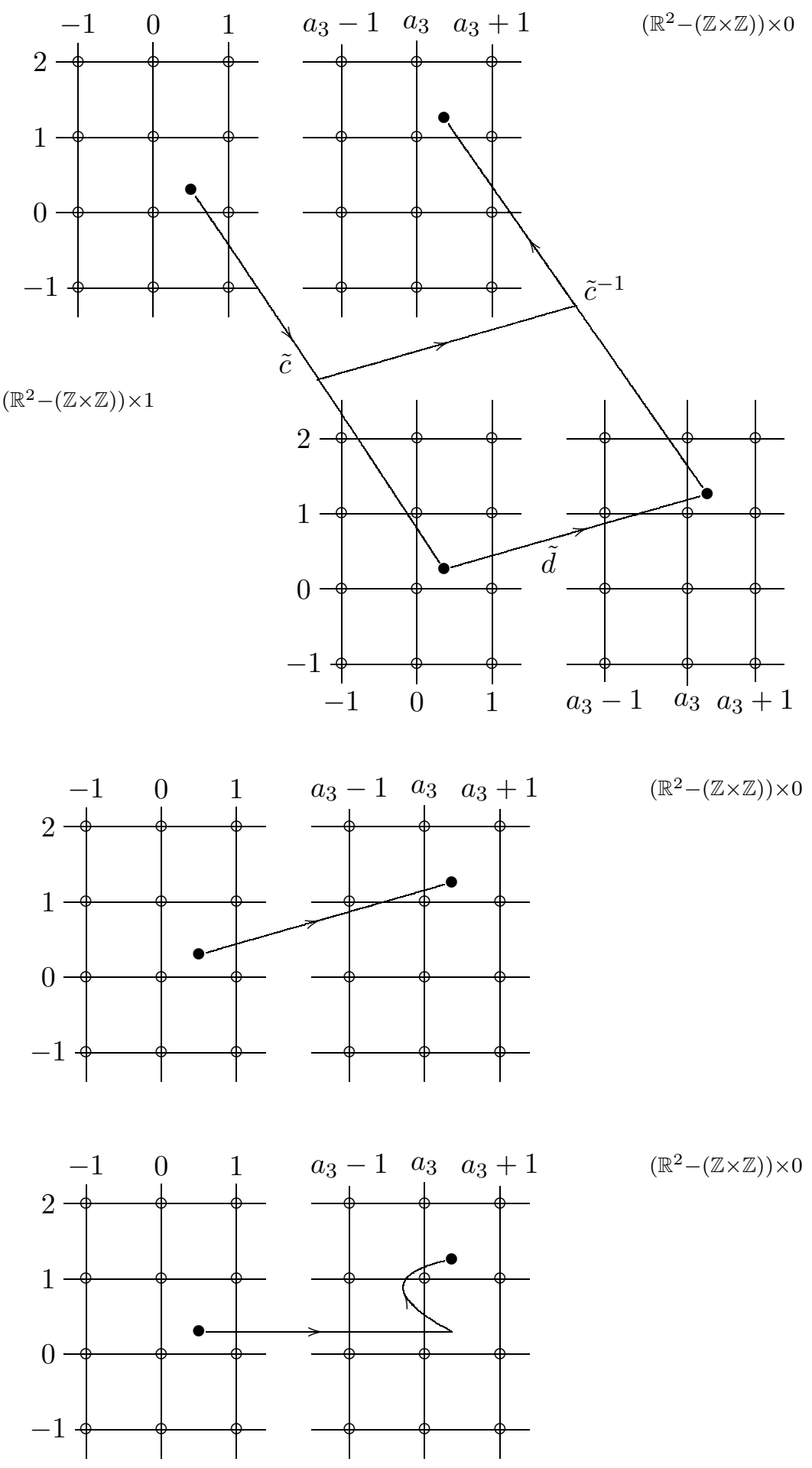


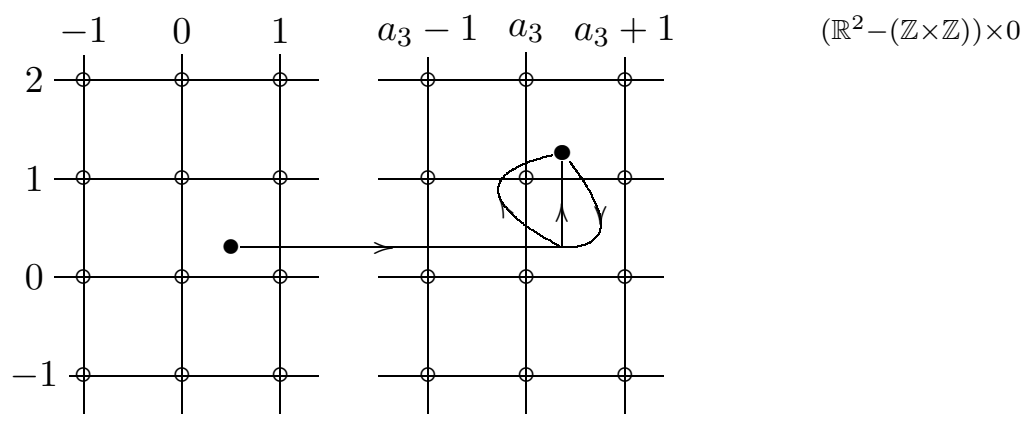

This last path when projected on $M A-S^{1}$ gives $e^{a_{3}} d W^{-1}$ and therefore $\tilde{c} \tilde{c} \tilde{c}^{-1}=e^{a_{3}} d W^{-1}$.

(3) In fact,

$$
\begin{aligned}
& \left(p_{2} \circ h \circ(1, f)\right)_{\#}(a)=\left(p_{2} \circ h\right)_{\#}(1, f)_{\#}(a)=\left(p_{2} \circ h\right)_{\#}\left(a, f_{\#}(a)\right) \\
& =\left(p_{2} \circ h\right)_{\#}\left(a,(f \mid T)_{\#}(a)\right)=\left(p_{2} \circ h\right)_{\#}([a(t)],[(f \mid T)(a(t))]) \\
& =\left(p_{2} \circ h\right)_{\#}\left(\left[\left\langle\left[\begin{array}{l}
t \\
0
\end{array}\right], 0\right\rangle\right],\left[(f \mid T)\left(\left\langle\left[\begin{array}{l}
t \\
0
\end{array}\right], 0\right\rangle\right)\right]\right) \\
& =\left(p_{2} \circ h\right)_{\#}\left(\left[\left\langle\left[\begin{array}{c}
t \\
0
\end{array}\right], 0\right\rangle\right],\left[\left\langle\left[B\left(\begin{array}{c}
t \\
0
\end{array}\right)+\left(\begin{array}{c}
q \\
q
\end{array}\right)\right], 0\right\rangle\right]\right) \\
& =\left[\left(p_{2} \circ h\right)\left(\left\langle\left[\begin{array}{l}
t \\
0
\end{array}\right], 0\right\rangle,\left\langle\left[B\left(\begin{array}{l}
t \\
0
\end{array}\right)+\left(\begin{array}{l}
q \\
q
\end{array}\right)\right], 0\right\rangle\right)\right] \\
& =\left[\left(p_{2}\right)\left(\left\langle\left[\begin{array}{l}
t \\
0
\end{array}\right], 0\right\rangle,\left\langle\left[B\left(\begin{array}{c}
t \\
0
\end{array}\right)+\left(\begin{array}{c}
q \\
q
\end{array}\right)-\left(\begin{array}{l}
t \\
0
\end{array}\right)\right], 0\right\rangle\right)\right] \\
& =\left[\left\langle\left[B\left(\begin{array}{c}
t \\
0
\end{array}\right)+\left(\begin{array}{l}
q \\
q
\end{array}\right)-\left(\begin{array}{l}
t \\
0
\end{array}\right)\right], 0\right\rangle\right] \\
& =\left[\left\langle\left[\left(\begin{array}{c}
\left(b_{1}-1\right) t \\
b_{2} t
\end{array}\right)+\left(\begin{array}{l}
q \\
q
\end{array}\right)\right], 0\right\rangle\right] \\
& =\left[\left\langle\left[\begin{array}{c}
q+\left(b_{1}-1\right) t \\
q
\end{array}\right], 0\right\rangle\right] \cdot\left[\left\langle\left[\begin{array}{c}
q \\
b_{2} t
\end{array}\right], 0\right\rangle\right]=\bar{e}^{\left(b_{1}-1\right)} \bar{d}^{b_{2}} .
\end{aligned}
$$

Similarly we argue for $b$ and $c_{0}$.

(4) The proof is trivial.

(5) First we observe that if $\Gamma(a)=x$ then $i_{\#}(x)=i_{\#} \circ \Gamma(a)=\left(p_{2} \circ\right.$ $h \circ(1, f))_{\#}(a)=\bar{e}^{\left(b_{1}-1\right)} \bar{d}^{b_{2}}$. On the other hand, $i_{\#}\left(e^{b_{1}-1} d^{b_{2}}\right)=\bar{e}^{\left(b_{1}-1\right)} \bar{d}^{b_{2}}$. Therefore $x d^{-b_{2}} e^{1-b_{1}}=Z_{1}$, where $Z_{1} \in \pi_{1} \mathcal{F}\left(M A-S^{1}\right) \cong \pi_{2}(T, T-1)=$ $\operatorname{ker}\left(\pi_{1}(T-1) \rightarrow \pi_{1}(T)\right)$. Similarly we argue for $b$ and $c_{0}$. Now the equations

$$
\left\{\begin{array}{l}
{[\Gamma(a), \Gamma(b)]=1,} \\
\Gamma\left(c_{0}\right) \Gamma(a) \Gamma\left(c_{0}^{-1}\right)=\Gamma\left(a^{a_{1}} b^{a_{2}}\right), \\
\Gamma\left(c_{0}\right) \Gamma(b) \Gamma\left(c_{0}^{-1}\right)=\Gamma\left(a^{a_{3}} b^{a_{4}}\right)
\end{array}\right.
$$

follow from the relations on $\pi_{1}(M A)$.

Proposition 2.3 (Case I). Let $f: M A \rightarrow M A$ be a map over $S^{1}$ where $f$ corresponds to case $I$ where the matrix $B$ is the identity. Then $f$ can always be deformed to a fixed point free map by a fiberwise homotopy. 
Proof. Define a lift for $\left(p_{2} \circ h \circ(1, f)\right)_{\#}$ by

$$
\Gamma(a)=1, \quad \Gamma(b)=1, \quad \Gamma\left(c_{0}\right)=e^{c_{1}} d^{c_{2}} c .
$$

Now we derive a necessary algebraic condition for $f$ to be deformable to a fixed point free map.

Proposition 2.4. If $f: M A \rightarrow M A$ corresponds to the remaining cases other than $I$ and $f$ can be deformed to a fixed point free map by a fiberwise homotopy, then $Z_{1}=1$. In this case in order to have the homomorphism $\Gamma$ it is necessary and sufficient to solve the equation

$$
\Gamma\left(c_{0}\right) \Gamma(b) \Gamma\left(c_{0}^{-1}\right)=\Gamma\left(a^{a_{3}} b^{a_{4}}\right)=\Gamma\left(a^{a_{3}}\right) \Gamma\left(b^{a_{4}}\right)=\Gamma\left(b^{a_{4}}\right) .
$$

Proof. In the remaining cases, since $\left(b_{1}-1, b_{2}\right)=(0,0)$ and $\left(b_{3}, b_{4}-1\right) \neq$ $(0,0)$, we have $\Gamma(a)=Z_{1}$. But by Theorem 2.2(5), $\left[Z_{1}, \Gamma(b)\right]=1$. If $Z_{1} \neq 1$ then we must have $Z_{1}=u^{\alpha}$ and $\Gamma(b)=u^{\beta}$, where $u \in \pi_{2}(T, T-1)$. But this is impossible, since $i_{\pi} \circ \Gamma(b)=\left(p_{2} \circ h \circ(1, f)\right)_{\pi}(b)=\bar{e}^{b_{3}} \bar{d}^{\left(b_{4}-1\right)} \neq 1$. On the other hand, $i_{\pi} \circ \Gamma(b)=i_{\pi}\left(u^{\beta}\right)=1$ since $u \in \pi_{2}(T, T-1)$. Therefore $Z_{1}=1$; the second part is immediate.

\section{GENERALITIES AND PROPERTIES OF THE MAIN EQUATION}

In this section we first write in a more explicit form the main equation given by Proposition 2.4, interpreted as an equation in the free group $\pi_{2}(T, T-1)$. We derive some general results which are useful in solving the equation. Then we study the main equation in the abelianization of $\pi_{2}(T, T-1)$. We derive a necessary condition for the existence of a solution.

3.1. Main equation. Consider the equation given by Proposition 2.4. Let $E, D$ be any elements such that $j_{\#}(E)=j_{\#}\left(e^{c_{1}} d^{c_{2}}\right)$ and $j_{\#}(D)=$ $j_{\#}\left(e^{b_{3}} d^{b_{4}-1}\right)$. Then we have:

Proposition 3.1. Let $E$ and $D$ as above. Then the equation given by Proposition 2.4 can be written either

(1) in the form

$$
Z_{3} E c Z_{2} D c^{-1} E^{-1} Z_{3}^{-1}=\left(Z_{2} D\right)^{a_{4}}, \text { or }
$$

(2) in the form

$$
\begin{aligned}
& Z_{3} \cdot E c Z_{2} c^{-1} E^{-1} \cdot E\left(c D c^{-1} D^{-a_{4}}\right) E^{-1} \cdot\left[E, D^{a_{4}}\right] \\
& \cdot D^{a_{4}} Z_{3}^{-1} D^{-a_{4}} \cdot D^{\left(a_{4}-1\right) / 2} Z_{2}^{-a_{4}} D^{\left(1-a_{4}\right) / 2}=1 .
\end{aligned}
$$

Furthermore, $c D c^{-1} D^{-a_{4}} \in \operatorname{ker} j_{\#}$ and the existence of a solution of the above equation is independent of the choices of $E$ and $D$, for $Z_{2}, Z_{3} \in \operatorname{ker} j_{\#}$.

Proof. (1) As $j_{\#}(E)=j_{\#}\left(e^{c_{1}} d^{c_{2}}\right)$ and $j_{\#}(D)=j_{\#}\left(e^{b_{3}} d^{b_{4}-1}\right)$, there exist $\alpha_{E}, \alpha_{D} \in \operatorname{ker} j_{\#}$ so that $e^{c_{1}} d^{c_{2}}=\alpha_{E} E$ and $e^{b_{3}} d^{b_{4}-1}=\alpha_{D} D$. Now the 
equation

$$
\Gamma\left(c_{0}\right) \Gamma(b) \Gamma\left(c_{0}^{-1}\right)=\Gamma\left(b^{a_{4}}\right)
$$

is

$$
Z_{3} e^{c_{1}} d^{c_{2}} c Z_{2} e^{b_{3}} d^{b_{4}-1} c^{-1} d^{-c_{2}} e^{-c_{1}} Z_{3}^{-1}=\left(Z_{2} e^{b_{3}} d^{b_{4}-1}\right)^{a_{4}} .
$$

Substituting $e^{c_{1}} d^{c_{2}}=\alpha_{E} E$ and $e^{b_{3}} d^{b_{4}-1}=\alpha_{D} D$ we obtain

$$
Z_{3} \alpha E c Z_{2} \alpha_{D} D c^{-1} E^{-1} \alpha^{-1} Z_{3}^{-1}=\left(Z_{2} \alpha_{D} D\right)^{a_{4}} \text {. }
$$

For simplicity, we denote $Z_{3} \alpha_{E}$ and $Z_{2} \alpha_{D}$ again by $Z_{3}$ and $Z_{2}$ and so we obtain the equation

$$
Z_{3} E c Z_{2} D c^{-1} E^{-1} Z_{3}^{-1}=\left(Z_{2} D\right)^{a_{4}} .
$$

(2) The equation above is the same as

$$
Z_{3} E c Z_{2} c^{-1} E^{-1} E c D c^{-1} D^{-a_{4}} E^{-1} E D^{a_{4}} E^{-1} D^{-a_{4}} D^{a_{4}} Z_{3}^{-1}\left(Z_{2} D\right)^{-a_{4}}=1
$$

and therefore

$$
Z_{3} \cdot E c Z_{2} c^{-1} E^{-1} \cdot E\left(c D c^{-1} D^{-a_{4}}\right) E^{-1} \cdot\left[E, D^{a_{4}}\right] \cdot D^{a_{4}} Z_{3}^{-1}\left(Z_{2} D\right)^{-a_{4}}=1 .
$$

Now it is sufficient to observe that

$$
\left(Z_{2} D\right)^{-a_{4}}=D^{-a_{4}} \cdot D^{\left(a_{4}-1\right) / 2} Z_{2}^{-a_{4}} D^{\left(1-a_{4}\right) / 2}
$$

since $a_{4}= \pm 1$.

To prove that $c D c^{-1} D^{-a_{4}} \in \operatorname{ker} j_{\#}$ it suffices to see that $j_{\#}\left(c D c^{-1} D^{-a_{4}}\right)$ $=c j_{\#}(D) c^{-1} j_{\#}\left(D^{-a_{4}}\right)=0$, where the last equality is obtained by using the action of $c$ and the fact that $\pi_{1}(T)$ is abelian.

For the last part observe that any two choices of either $E$ 's or $D$ 's differ by elements of ker $j_{\#}$. So, the equation given by Proposition 3.1 has a solution for one choice if and only if it has a solution for the other choice, and the result follows.

Motivated by the proposition above we make:

Definition 3.2. An input datum for the main equation given by Proposition 2.4 is a quadruple $\left(b_{3}, b_{4}, c_{1}, c_{2}\right)$.

From Proposition 3.1 we see that the existence of a solution of the main equation depends only in the input. Also, observe that the input defines the $\operatorname{map} f$.

By ||$_{e},||_{d}: \pi_{1}(T-1) \rightarrow \mathbb{Z}$ we denote the homomorphisms which map a word $w$ to the sum of the exponents of the generator $e$ and the sum of the exponents of $d$, respectively.

The next theorem shows that, for a fixed group $\pi_{1}(M A)$, the existence of a solution for one equation implies the existence of a solution of other equations. This is done in terms of the inputs $\left(b_{3}, b_{4}, c_{1}, c_{2}\right)$. More precisely: 
Theorem 3.3. Let $A$ and $B$ be fixed.

(1) The equation given by Proposition 3.1 has a solution for given $E, D$ if and only if it has a solution for $w E c w^{-1} c^{-1}, w D w^{-1}$.

(2) The equations given in (1) have the coordinates $c_{1}, c_{2}$ of the input related as follows:

$$
\left\{\begin{array}{l}
\left|w E c w^{-1} c^{-1}\right|_{e}=|E|_{e}+\left|w c w^{-1} c^{-1}\right|_{e} \\
\left|w E c w^{-1} c^{-1}\right|_{d}=|E|_{d}+\left|w c w^{-1} c^{-1}\right|_{d}
\end{array}\right.
$$

Proof. (1) In fact, there exists a lifting homomorphism $\Gamma$ in diagram (2.2) for the input data $(A, B, E, D)$ if and only if there exist $Z_{2}, Z_{3} \in$ $\pi_{2}(T, T-1)$ so that

$$
Z_{3} E c Z_{2} D c^{-1} E^{-1} Z_{3}^{-1}=\left(Z_{2} D\right)^{a_{4}}
$$

if and only if for all $w \in \pi_{1}(T-1)$,

$$
\begin{aligned}
w Z_{3} E c Z_{2} D c^{-1} E^{-1} Z_{3}^{-1} w^{-1} & =w\left(Z_{2} D\right)^{a_{4}} w^{-1}=\left(w Z_{2} D w^{-1}\right)^{a_{4}} \\
& =\left(w Z_{2} w^{-1} w D w^{-1}\right)^{a_{4}} .
\end{aligned}
$$

Therefore

$$
\begin{array}{r}
w Z_{3} w^{-1} w E c w^{-1} c^{-1} c w Z_{2} w^{-1} w D w^{-1} w c^{-1} E^{-1} w^{-1} w Z_{3}^{-1} w^{-1} \\
=\left(w Z_{2} w^{-1} w D w^{-1}\right)^{a_{4}}
\end{array}
$$

if and only if there exist $\bar{Z}_{2}=w Z_{2} w^{-1}, \bar{Z}_{3}=w Z_{3} w^{-1} \in \pi_{2}(T, T-1)$ so that

$$
\bar{Z}_{3} \cdot w E c w^{-1} c^{-1} c \bar{Z}_{2} w D w^{-1} w c^{-1} E^{-1} w^{-1} \bar{Z}_{3}^{-1}=\left(\bar{Z}_{2} w D w^{-1}\right)^{a_{4}}
$$

if and only if there exists a lifting homomorphism $\Gamma$ in diagram (2.2) for the input data $\left(A, B, w E c w^{-1} c^{-1}, w D w^{-1}\right)$.

(2) In fact, we have

$$
\begin{aligned}
\left|w E c w^{-1} c^{-1}\right|_{e} & =\left|w E w^{-1} w c w^{-1} c^{-1}\right|_{e} \\
& =\left|w E w^{-1}\right|_{e}+\left|w c w^{-1} c^{-1}\right|_{e} \\
& =|E|_{e}+\left|w c w^{-1} c^{-1}\right|_{e} .
\end{aligned}
$$

The other case is similar.

Corollary 3.4. Let $H$ be the image of the homomorphism $\pi_{1}(T-1)$ $\rightarrow \mathbb{Z} \oplus \mathbb{Z}$ which maps $\alpha$ to the pair $\left(\left|c \alpha c^{-1} \alpha^{-1}\right|_{e},\left|c \alpha c^{-1} \alpha^{-1}\right|_{d}\right)$. If two input data $\left(b_{3}, b_{4}, c_{1}, c_{2}\right)$ and $\left(b_{3}, b_{4}, c_{1}^{\prime}, c_{2}^{\prime}\right)$ have the property that the pairs $\left(c_{1}, c_{2}\right)$ and $\left(c_{1}^{\prime}, c_{2}^{\prime}\right)$ belong to the same equivalence class in $\mathbb{Z} \oplus \mathbb{Z} / H$, then there is a solution for the equation with one of the inputs if and only if there is a solution for the other.

Proof. Suppose that for the input datum $\left(b_{3}, b_{4}, c_{1}, c_{2}\right)$ there is a solution, and $\left(b_{3}, b_{4}, c_{1}^{\prime}, c_{2}^{\prime}\right)$ is another input datum so that $\left(c_{1}, c_{2}\right)$ and $\left(c_{1}^{\prime}, c_{2}^{\prime}\right)$ belong 
to the same equivalence class in $\mathbb{Z} \oplus \mathbb{Z} / H$. Then there exists $w \in \pi_{1}(T-1)$ so that

$$
\left(c_{1}^{\prime}, c_{2}^{\prime}\right)-\left(c_{1}, c_{2}\right)=\left(\left|c w c^{-1} w^{-1}\right|_{e},\left|c w c^{-1} w^{-1}\right|_{d}\right) .
$$

Let $E$ be such that $\left(|E|_{e},|E|_{d}\right)=\left(c_{1}, c_{2}\right)$. Then the equation has a solution for a suitable $D$. Define $E^{\prime}=w E c w^{-1} c^{-1}$. By Theorem 3.3(1), the main equation also has a solution for $E^{\prime}$. Also by Theorem 3.3(2),

$$
\left(\left|E^{\prime}\right|_{e},\left|E^{\prime}\right|_{d}\right)=\left(\left|w E c w^{-1} c^{-1}\right|_{e},\left|w E c w^{-1} c^{-1}\right|_{d}\right) .
$$

Therefore, for the input $\left(b_{3}, b_{4}, c_{1}^{\prime}, c_{2}^{\prime}\right)$ we also have a solution and the result follows.

Remark 3.1. Let $A$ and $B$ be given, and $\mathcal{C}$ be a set of representatives of the equivalence classes of $\mathbb{Z} \oplus \mathbb{Z} / H$. In order to analyze all the cases it suffices to analyze the equation for the set of inputs $\left(b_{3}, b_{4}, c_{1}, c_{2}\right)$ such that $\left(c_{1}, c_{2}\right)$ runs over the set $\mathcal{C}$.

3.2. Equation on the abelianization. Let $\pi_{2}=\pi_{2}(T, T-1)$ denote the kernel of the map $j_{\#}:\langle e, d\rangle=\pi_{1}(T-1) \rightarrow \pi_{1} T=\langle e, d:[e, d]=1\rangle$ and $B=[e, d]$. We will study the equation given by Proposition 2.4 on the abelianization $\left(\pi_{2}\right)_{\mathrm{ab}}=\pi_{2} /\left[\pi_{2}, \pi_{2}\right]$ of $\pi_{2}$ and also on some quotients of this group. Whenever the equation in one of these quotients has no solution, we can infer that the original equation has no solution. The group $\pi_{2}$ is isomorphic to $\pi_{1}(\mathcal{F})$, where $\mathcal{F} \rightarrow E(T-1) \rightarrow T$ is the fibration obtained by making the inclusion $T-1 \hookrightarrow T$ into a fibration. So the group $\pi_{1}(T)$ acts on $H_{1}(\mathcal{F})=\pi_{2} /\left[\pi_{2}, \pi_{2}\right]$.

Proposition 3.5. (1) We have

$$
H_{1}(\mathcal{F}) \cong \mathbb{Z} \pi_{1}(T) \cong \bigoplus_{x, y \in \mathbb{Z}} B_{e^{x}} d^{y}
$$

where $B_{e^{x} d^{y}}=B_{(x, y)}=\mathcal{A}\left(e^{x} d^{y}[e, d] d^{-y} e^{-x}\right)$ is a generator of a copy of $\mathbb{Z}$. Here $\mathcal{A}: \pi_{2}(T, T-1)=\pi_{2} \rightarrow \pi_{2} /\left[\pi_{2}, \pi_{2}\right]=\mathbb{Z} \pi_{1}(T)$ is the natural projection.

(2) If , by means of this isomorphism, an element of $H_{1}(\mathcal{F})$ corresponds to the generator $1_{w}$ of the copy $\mathbb{Z}$, indexed by an element $w \in \pi_{1}(T)$, then the action of $\alpha \in \pi_{1}(T)$ on $B_{w}$ is the generator of the copy of $\mathbb{Z}$ indexed by the product $\alpha w$, namely $B_{\alpha w}$.

Proof. Let $p: \mathbb{R}^{2} \rightarrow T$ be the universal covering map and let $\tilde{x}_{0}$ and $x_{0}$ be the base points of $\mathbb{R}^{2}-p^{-1}(1)$ and $T-1$, respectively.

From Remarks 1.1, $\pi_{1}(\mathcal{F}) \cong \pi_{2}\left(T, T-1 ; x_{0}\right)$.

Take $\alpha^{\prime} \in \pi_{1}\left(T-1, x_{0}\right)$ such that $j_{\#}\left(\alpha^{\prime}\right)=\alpha$, where $j_{\#}$ is the epimorphism induced by the inclusion $j: T-1 \rightarrow T$. We consider the following diagram: 


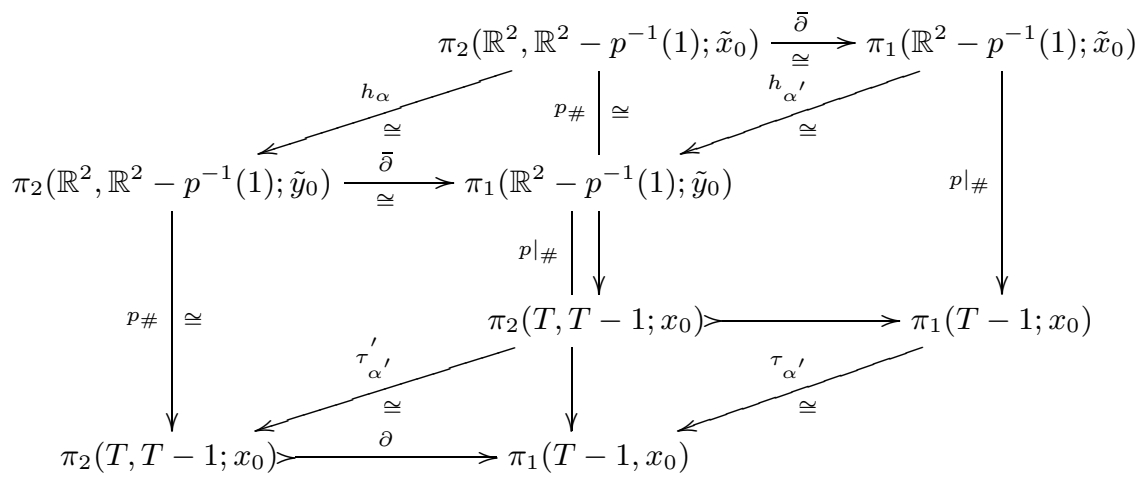

The map $h_{\alpha}$ is the deck transformation corresponding to $\alpha$ and $\tilde{y}_{0}=h_{\alpha}\left(\tilde{x}_{0}\right)$. The isomorphism $\tau_{\alpha^{\prime}}^{\prime}$ is the action of $\alpha^{\prime}$.

From these isomorphisms, we conclude that $H_{1}(\mathcal{F})$ is the abelianization of $\pi_{2}\left(\mathbb{R}^{2}, \mathbb{R}^{2}-p^{-1}(1) ; \tilde{x}_{0}\right) \cong \pi_{1}\left(\mathbb{R}^{2}-p^{-1}(1) ; \tilde{x}_{0}\right)$; therefore item (1) is proved.

Note that the left face of the diagram is not commutative, but if we look at this diagram on homology groups that face is commutative.

Geometrically, if we suppose $\alpha=e^{m} d^{n}$, then $h_{\alpha}$ translates a small circle around the point $(x, y) \in p^{-1}(1)$, which represents a generator $B_{(x, y)} \in$ $H_{1}\left(\mathbb{R}^{2}-p^{-1}(1)\right)$, to a circle around $(x+m, y+n)$. When we identify $B_{(x, y)}$ in $\mathbb{Z} \pi_{1}(T)$, this corresponds to the product $\alpha \cdot\left(e^{x} d^{y}\right)$ and (2) is proved.

We denote by || the homomorphism $\mathcal{E} \circ \mathcal{A}$ where $\mathcal{A}$ is the abelianization homomorphism and $\mathcal{E}: \mathbb{Z}\left(\pi_{1}(T)\right) \rightarrow \mathbb{Z}$ is the augmentation homomorphism, i.e. $\mathcal{E}\left(B_{w}\right)=1 \in \mathbb{Z}$ for all $w \in \pi_{1}(T)$.

Theorem 3.6. (1) $\left|\alpha Z \alpha^{-1}\right|=|Z|$ for all $\alpha \in \pi_{1}(T-1)$ and $Z \in$ $\pi_{2}(T, T-1)$.

(2) $\left|\left[e^{x_{1}} d^{y_{1}}, e^{x_{2}} d^{y_{2}}\right]\right|=\operatorname{det}\left[\begin{array}{ll}x_{1} & x_{2} \\ y_{1} & y_{2}\end{array}\right]$.

(3) $\left|\left[Z e^{x_{1}} d^{y_{1}}, W e^{x_{2}} d^{y_{2}}\right]\right|=\left|\left[e^{x_{1}} d^{y_{1}}, e^{x_{2}} d^{y_{2}}\right]\right|$ for all $Z, W \in \pi_{2}(T, T-1)$.

(4) If $c \in \pi_{1}\left(M A-S^{1}\right)$ as in diagram (2.2) then

$$
\left|c Z c^{-1}\right|=[\operatorname{sgn} \operatorname{det}(A)]|Z| \quad \text { for all } Z \in \pi_{2}(T, T-1) .
$$

Proof. (1) By definition $\left|\alpha Z \alpha^{-1}\right|=\mathcal{E} \circ \mathcal{A}\left(\alpha Z \alpha^{-1}\right)=\mathcal{E}\left(j_{\#}(\alpha) \cdot \mathcal{A}(Z)\right)$, where $j_{\#}: \pi_{1}(T-1) \rightarrow \pi_{1}(T)$. So, if $j_{\#}(\alpha)=e^{m} d^{n}$ and $\mathcal{A}(Z)=B_{\left(x_{1}, y_{1}\right)}^{t_{1}}$ then $j_{\#}(\alpha) \cdot \mathcal{A}(Z)=B_{\left(x_{1}+m, y_{1}+n\right)}^{t_{1}}$. Therefore,

$$
\left|\alpha Z \alpha^{-1}\right|=\mathcal{E}\left(j_{\#}(\alpha) \cdot \mathcal{A}(Z)\right)=\mathcal{E} \circ \mathcal{A}(Z)=|Z| .
$$

(2) We note that

(i) $\left|\left[e^{x_{1}} d^{y_{1}}, e^{x_{2}} d^{y_{2}}\right]\right|=\left|e^{x_{1}}\left[d^{y_{1}}, e^{x_{2}}\right] e^{-x_{1}} e^{x_{2}}\left[e^{x_{1}}, d^{y_{2}}\right] e^{-x_{2}}\right|$

$$
=\left|\left[d^{y_{1}}, e^{x_{2}}\right]\right|+\left|\left[e^{x_{1}}, d^{y_{2}}\right]\right| \text {. }
$$


(ii) From $\left[d^{y_{1}}, e^{x_{2}}\right]\left[e^{x_{2}}, d^{y_{1}}\right]=1$, we have $\left|\left[d^{y_{1}}, e^{x_{2}}\right]\right|=-\left|\left[e^{x_{2}}, d^{y_{1}}\right]\right|$.

(iii) From $\left[e^{x}, d^{-y}\right] d^{-y}\left[e^{x}, d^{y}\right] d^{y}=1$, we have $\left|\left[e^{x}, d^{-y}\right]\right|=-\left|\left[e^{x}, d^{y}\right]\right|$. Therefore, it is sufficient to prove $\left|\left[e^{x}, d^{y}\right]\right|=x y$ when $y \geq 0$. This follows by induction on $y$.

(3) We have

$$
\begin{aligned}
\left|\left[Z e^{x_{1}} d^{y_{1}}, W e^{x_{2}} d^{y_{2}}\right]\right| & \\
= & \left|Z e^{x_{1}} d^{y_{1}} W d^{-y_{1}} e^{-x_{1}}\left[e^{x_{1}} d^{y_{1}}, e^{x_{2}} d^{y_{2}}\right] e^{x_{2}} d^{y_{2}} Z^{-1} d^{-y_{2}} e^{-x_{2}} W^{-1}\right| \\
= & |Z|+\left|e^{x_{1}} d^{y_{1}} W d^{-y_{1}} e^{-x_{1}}\right|+\left|\left[e^{x_{1}} d^{y_{1}}, e^{x_{2}} d^{y_{2}}\right]\right| \\
& \quad+\left|e^{x_{2}} d^{y_{2}} Z^{-1} d^{-y_{2}} e^{-x_{2}}\right|-|W| \\
= & |Z|+|W|+\left|\left[e^{x_{1}} d^{y_{1}}, e^{x_{2}} d^{y_{2}}\right]\right|-|Z|-|W|=\left|\left[e^{x_{1}} d^{y_{1}}, e^{x_{2}} d^{y_{2}}\right]\right| .
\end{aligned}
$$

(4) We have $c B c^{-1}=c e c^{-1} c d c^{-1} c e^{-1} c^{-1} c d^{-1} c^{-1}$. We observe that in any case $c e c^{-1}=\alpha e^{\epsilon}$ and $c d c^{-1}=\beta e^{a_{3}} d^{\eta}$ with $\alpha, \beta \in \pi_{2}(T, T-1)$ and

$$
A=\left(\begin{array}{cc}
\epsilon & a_{3} \\
0 & \eta
\end{array}\right), \quad \text { where } \epsilon \eta= \pm 1 .
$$

It follows from (3) that $\left|c B c^{-1}\right|=\left|e^{a_{3}}\left[e^{\epsilon}, d^{\eta}\right] e^{-a_{3}}\right|=\operatorname{sgn} \operatorname{det} A$. In the general case, $Z$ can be written in the form

$$
Z=\prod_{i=1}^{r} \alpha_{i} B^{t_{i}} \alpha_{i}^{-1} .
$$

So, $c Z c^{-1}=\prod_{i=1}^{r} c \alpha_{i} c^{-1}\left(c B c^{-1}\right)^{t_{i}} c \alpha_{i}^{-1} c^{-1}$ and therefore

$$
\begin{aligned}
\left|c Z c^{-1}\right| & =\mathcal{E} \circ \mathcal{A}\left(c Z c^{-1}\right)=\mathcal{E}\left(\prod_{i=1}^{r} B_{j_{\#}\left(c \alpha_{i} c^{-1}\right)}^{\left|c B c^{-1}\right| t_{i}}\right) \\
& =\sum_{i=1}^{r}\left|c B c^{-1}\right| t_{i}=\left|c B c^{-1}\right| \sum_{i=1}^{r} t_{i}=\left|c B c^{-1}\right||Z| .
\end{aligned}
$$

Now we consider the equation given by Proposition 2.4. We will look at it in the abelianization of $\pi_{2}(T, T-1)$, which is $\mathbb{Z} \pi_{1}(T)$, and in a quotient of $\mathbb{Z} \pi_{1}(T)$, namely $\mathbb{Z} \tilde{H}$, where $\tilde{H}=\mathbb{Z} \oplus \mathbb{Z} /\left\langle\left(c_{1}, c_{2}\right),\left(b_{3}, b_{4}-1\right)\right\rangle$. Denote by $\bar{x}$ the image of $x \in \pi_{2}(T, T-1)$ either in $\mathbb{Z} \pi_{1}(T)$ or in $\mathbb{Z} \tilde{H}$. Then we have:

Proposition 3.7. Let $E$ and $D$ as in Proposition 3.1. Then the main equation given in Proposition 2.4 is:

(1) of the form

$$
\begin{aligned}
& Z_{3} \cdot E c Z_{2} c^{-1} E^{-1} \cdot \overline{E\left(c D c^{-1} D^{-a_{4}}\right) E^{-1}} \cdot \overline{\left[E, D^{a_{4}}\right]} \\
& \cdot D^{a_{4}} Z_{3}^{-1} D^{-a_{4}} \cdot D^{\left(a_{4}-1\right) / 2} Z_{2}^{-a_{4}} D^{\left(1-a_{4}\right) / 2}=1
\end{aligned}
$$

in the abelianization, where $Z_{2}, Z_{3} \in \mathbb{Z} \pi_{1}(T)$, 
(2) of the form

$$
c Z_{2} c^{-1} \cdot \overline{c D c^{-1} D^{-a_{4}}} \cdot \overline{\left[E, D^{a_{4}}\right]} \cdot Z_{2}^{-a_{4}}=1
$$

in $\mathbb{Z} \tilde{H}$, where $Z_{2} \in \mathbb{Z} \tilde{H}$.

Proof. In $\mathbb{Z} \tilde{H}$, where $\tilde{H}=\mathbb{Z} \oplus \mathbb{Z} /\left\langle\left(c_{1}, c_{2}\right),\left(b_{3}, b_{4}-1\right)\right\rangle$, the equation

$Z_{3} \cdot E c Z_{2} c^{-1} E^{-1} \cdot \overline{E\left(c D c^{-1} D^{-a_{4}}\right) E^{-1}} \cdot \overline{\left[E, D^{a_{4}}\right]}$

$$
\text { . } D^{a_{4}} Z_{3}^{-1} D^{-a_{4}} \cdot D^{\left(a_{4}-1\right) / 2} Z_{2}^{-a_{4}} D^{\left(1-a_{4}\right) / 2}=1
$$

reduces to $c Z_{2} c^{-1} \cdot \overline{c D c^{-1} D^{-a_{4}}} \cdot \overline{\left[E, D^{a_{4}}\right]} \cdot Z_{2}^{-a_{4}}=1$, since $\mathbb{Z} \tilde{H}$ is abelian and in this quotient

$$
\begin{array}{cl}
E c Z_{2} c^{-1} E^{-1}=c Z_{2} c^{-1}, & \overline{E\left(c D c^{-1} D^{-a_{4}}\right) E^{-1}}=\overline{c D c^{-1} D^{-a_{4}}}, \\
D^{a_{4}} Z_{3}^{-1} D^{-a_{4}}=Z_{3}^{-1}, & D^{\left(a_{4}-1\right) / 2} Z_{2}^{-a_{4}} D^{\left(1-a_{4}\right) / 2}=Z_{2}^{-a_{4}} .
\end{array}
$$

By applying the homomorphism | | to the left-hand side of the equation given in Proposition 3.7(2) we obtain:

Corollary 3.8 .

$$
[\operatorname{sgn} \operatorname{det}(A)]\left|Z_{2}\right|+\left|c D c^{-1} D^{-a_{4}}\right|+\left|\left[E, D^{a_{4}}\right]\right|+\left|Z_{2}^{-a_{4}}\right|=0 .
$$

Proof. By Theorem 3.6(4), we have $\left|c Z_{2} c^{-1}\right|=[\operatorname{sgn} \operatorname{det}(A)]\left|Z_{2}\right|$ and the result follows.

\section{THE MAIN RESULT: SOLUTIONS OF THE MAIN EQUATION}

In this section we prove the main result of this work:

TheOREM 4.1. A fiber-preserving map $f: M(\phi) \rightarrow M(\phi)$ can be deformed to a fixed point free map by a homotopy over $S^{1}$ if and only if one of the cases below holds:

(1) $M(\phi)$ is as in case $I$ and $f$ is arbitrary.

(2) $M(\phi)$ is as in one of cases II, III, IV and $c_{1}\left(b_{4}-1\right)-c_{2} b_{3}=0$.

(3) $M(\phi)$ is as in case $V$ and

$$
b_{4}\left(b_{3}+1\right)+c_{1}\left(1-b_{4}\right)+c_{2} b_{3}-1 \equiv 0 \bmod 2
$$

except in the cases listed in the table below:

\begin{tabular}{ccccc}
\hline$a_{3}$ & $\left(b_{3}, b_{4}\right)$ & $\left(c_{1}, c_{2}\right)$ & $E$ & $D$ \\
\hline $2 r>0$ & $(2 s, 1)$ & $\equiv(0,0)$ & 1 & $e^{2 s}$ \\
$2 r<0$ & $(2 s, 1)$ & $\equiv(0,0)$ & {$\left[d^{-1}, e^{-1}\right]$} & $e^{2 s}$ \\
$2 r+1>0$ & $(2 s, 1)$ & $\equiv(0,0)$ & 1 & $e^{2 s}$ \\
$2 r+1<0$ & $(2 s, 1)$ & $\equiv(0,0)$ & {$\left[d^{-1}, e^{-1}\right]$} & $e^{2 s}$ \\
0 & $(2 s, 1+2 k)$ & $\equiv(0,0)$ & 1 & $d^{k} e^{2 s} d^{k}$ \\
\hline
\end{tabular}


(4) $M(\phi)$ is as in case VI and either

(I) $a_{3}$ is even and

$$
\left(b_{4}-1\right)\left(c_{1}-1-c_{2} r\right)=0 \bmod 2
$$

except when $b_{4}-1=2^{n_{1}} p_{1}$, where $p_{1} \geq 1$ is odd, and $c_{2}=2^{n_{2}} p_{2}$, where $p_{2}$ is odd, $1<n_{1} \leq n_{2}$ and $c_{1}-r c_{2} \equiv 0 \bmod 2$, or

(II) $a_{3}$ is odd and

$$
k\left(1+c_{2}\right)=0 \bmod 2
$$

except when $\left(b_{4}-1\right) / L=2 p+1$ and $c_{2}=2 q$, where $L=$ $\operatorname{gcd}\left(b_{4}-1, c_{2}\right)$.

The rest of this section is devoted to the proof of this result. We briefly describe our approach to decide if an equation has a solution or not.

1) First we compute the necessary condition given by Corollary 3.8 and the set of equivalence classes as defined by Corollary 3.4.

2) Then we choose a set $\mathcal{C}$ of representatives of the equivalence classes in $\mathbb{Z} \oplus \mathbb{Z} / H$ given by Corollary 3.4. For some $\left(c_{1}, c_{2}\right) \in \mathcal{C}$ we find elements $E, D$ which correspond to the input $\left(b_{3}, b_{4}, c_{1}, c_{2}\right)$ and satisfy

$$
E c D c^{-1} E^{-1} D^{-a_{4}}=1 \text {. }
$$

This tells us that the equation given by Proposition 3.7(1), with $E, D$ chosen as above, admits the trivial solution $Z_{2}=Z_{3}=1$, and allows us to write a sufficient condition, in terms of the data, which guarantees the existence of a solution.

3) For some classes $\left(c_{1}, c_{2}\right) \in \mathcal{C}$ we show that there is no solution by looking at the main equation either in $\mathbb{Z} \pi_{1}(T)$, which is the abelianization of $\pi_{2}(T, T-1)$, or in $\mathbb{Z} \tilde{H}$, where $\tilde{H}$ is $\mathbb{Z} \oplus \mathbb{Z} / H$ and $H$ contains the subgroup $\left\langle\left(c_{1}, c_{2}\right),\left(b_{3}, b_{4}-1\right)\right\rangle$. Then we use Corollary 3.4 and Proposition 3.7.

CAse I: It was solved in Proposition 2.3.

CASE II: $A^{1}=\left(\begin{array}{ll}1 & 0 \\ 0 & 1\end{array}\right)$ and $B^{1}=\left(\begin{array}{ll}1 & b_{3} \\ 0 & b_{4}\end{array}\right)$. The equation to be solved is

$$
Z_{3} E c Z_{2} c^{-1} E^{-1} E\left(c D c^{-1} D^{-1}\right) E^{-1}[E, D] D Z_{3}{ }^{-1} D^{-1} Z_{2}{ }^{-1}=1 .
$$

(1) The condition given by Corollary 3.8, called the necessary condition, is

$$
\left|c D c^{-1} D^{-1}\right|+|[E, D]|=0,
$$

but for every $D$ we have $c D c^{-1} D^{-1}=1$, so

$$
|[E, D]|=0=\operatorname{det}\left(\begin{array}{cc}
c_{1} & b_{3} \\
c_{2} & b_{4}-1
\end{array}\right) .
$$


(2) The sufficient condition is

$$
\operatorname{det}\left(\begin{array}{cc}
c_{1} & b_{3} \\
c_{2} & b_{4}-1
\end{array}\right)=0
$$

We consider $L=\operatorname{gcd}\left(b_{3}, b_{4}-1\right)$ and let $\left(k_{1}, k_{2}\right)$ be so that $\left(b_{3}, b_{4}-1\right)=$ $L\left(k_{1}, k_{2}\right)$. If the above determinant is 0 then there exists $t \in \mathbb{Z}$ that $\left(c_{1}, c_{2}\right)=$ $t\left(k_{1}, k_{2}\right)$. We take $E=v^{t}$ and $D=v^{L}$, where $v=e^{k_{1}} d^{k_{2}}$, and it is easy to verify that $[E, D]=1$ and $c D c^{-1} D^{-1}=1$, so the equation admits the trivial solution $Z_{2}=Z_{3}=1$.

CASE III: $A^{1}=\left(\begin{array}{cc}1 & a_{3} \\ 0 & 1\end{array}\right)$ and $B^{1}=\left(\begin{array}{cc}1 & b_{3} \\ 0 & 1\end{array}\right)$ with $b_{3} \neq 0$. The equation to be solved is

$$
Z_{3} E c Z_{2} c^{-1} E^{-1} E\left(c D c^{-1} D^{-1}\right) E^{-1}[E, D] D Z_{3}^{-1} D^{-1} Z_{2}{ }^{-1}=1 .
$$

(1) The necessary condition is

$$
\left|c D c^{-1} D^{-1}\right|+|[E, D]|=0 .
$$

To compute $\left|c D c^{-1} D^{-1}\right|$, we take $D=e^{b_{3}}$; then $c D c^{-1} D^{-1}=1$. So, the relation above becomes

$$
|[E, D]|=0=\operatorname{det}\left(\begin{array}{cc}
c_{1} & b_{3} \\
c_{2} & 0
\end{array}\right)=-c_{2} b_{3}, \quad b_{3} \neq 0,
$$

which implies $c_{2}=0$.

(2) The sufficient condition is $c_{2}=0$. If this condition is satisfied we take $E=e^{c_{1}}$ and $D=e^{b_{3}}$ and so $[E, D]=1$. Therefore, the equation admits the trivial solution $Z_{2}=Z_{3}=1$.

CASE IV: $A^{1}=\left(\begin{array}{cc}1 & a_{3} \\ 0 & -1\end{array}\right), B^{1}=\left(\begin{array}{ll}1 & b_{3} \\ 0 & b_{4}\end{array}\right)$ and $a_{3}\left(b_{4}-1\right)=-2 b_{3}$ with $b_{4}-1$ $\neq 0$. The equation to be solved is

$$
Z_{3} E c Z_{2} c^{-1} E^{-1}\left(E\left(c D c^{-1} D\right) E^{-1}\left[E, D^{-1}\right]\right) D^{-1} Z_{3}{ }^{-1} D D^{-1} Z_{2} D=1 .
$$

(1a) The necessary condition is

$$
\left|c D c^{-1} D\right|+\left|\left[E, D^{-1}\right]\right|=0 .
$$

In order to calculate this condition, first we consider $a_{3} \geq-1$. Since $b_{3}=$ $-a_{3}\left(b_{4}-1\right) / 2,2$ divides either $a_{3}$ or $b_{4}-1$. If 2 divides $a_{3}$ consider $v=$ $e^{-a_{3} / 2} d$, otherwise 2 must divide $b_{4}-1$ and consider $v=d e^{-a_{3}} d$. From the presentation of the group for $a_{3} \geq-1$ we have $c v c^{-1}=v^{-1}$.

Therefore, if $D=v^{b_{4}-1}$ then $c D c^{-1} D=1$ so $\left|c D c^{-1} D\right|=0$.

Let $a_{3} \leq-2$ and consider the presentation which corresponds to this case. If we set $\beta=e^{-1} d^{-1} e$, then the presentation is given by

$$
\left\langle e, d, c: c e c^{-1}=\beta e \beta^{-1}, c d c^{-1}=\beta e^{a_{3}} d^{-1} \beta^{-1}\right\rangle .
$$

Take $v$ as in the previous case; similar calculations show that

$$
c v c^{-1}=\beta d v^{-1} d^{-1} \beta^{-1}=\left[e^{-1}, d^{-1}\right] v^{-1}\left[d^{-1}, e^{-1}\right] .
$$


So, if we take $D=v^{b_{4}-1}$ or $D=v^{\left(b_{4}-1\right) / 2}$, then $c D c^{-1} D=\left[\left[e^{-1}, d^{-1}\right], D\right]$ and therefore $\left|c D c^{-1} D\right|=0$. Thus, the necessary condition is $\left|\left[E, D^{-1}\right]\right|=$ $0=c_{1}\left(b_{4}-1\right)+c_{2} b_{3}$.

(1b) The image of the map $\pi_{1}(T-1)=\langle e, d\rangle \rightarrow \mathbb{Z} \oplus \mathbb{Z}$ given by

$$
\alpha \mapsto\left(\left|\alpha c \alpha^{-1} c^{-1}\right|_{e},\left|\alpha c \alpha^{-1} c^{-1}\right|_{d}\right)
$$

is denoted by $\operatorname{im}\left(||_{e},||_{d}\right)$. We have $e \mapsto(0,0), d \mapsto\left(-a_{3}, 2\right)$, so

$$
\frac{\mathbb{Z} \oplus \mathbb{Z}}{\operatorname{im}\left(||_{e},||_{d}\right)}=\frac{\mathbb{Z} \oplus \mathbb{Z}}{\left\langle\left(a_{3},-2\right)\right\rangle}
$$

(1c) We have

$$
\frac{\mathbb{Z} \oplus \mathbb{Z}}{\left\langle\left(a_{3},-2\right)\right\rangle}= \begin{cases}\frac{\left\langle\left(a_{3},-2\right),(-r, 1)\right\rangle}{\left\langle\left(a_{3},-2\right)\right\rangle} \cong \mathbb{Z} & \text { if } a_{3}=2 r+1, \\ \frac{\langle(-1,0),(r,-1)\rangle}{\langle(2 r,-2)\rangle} \cong \mathbb{Z} \oplus \mathbb{Z}_{2} & \text { if } a_{3}=2 r .\end{cases}
$$

(2a) The sufficient condition is $\left|\left[E, D^{-1}\right]\right|=0=c_{1}\left(1-b_{4}\right)+c_{2} b_{3}$. We are going to prove this in the steps that follow.

(2b) If $a_{3}=2 r+1$ then a complete set of representatives of $E$ is given by elements of the form $(0, y)$, where the coordinates are relative to the base $\left(\left(a_{3},-2\right),(-r, 1)\right)$ so $\left(c_{1}, c_{2}\right)=(-y r, y)$.

These values must satisfy the necessary condition $c_{1}\left(1-b_{4}\right)+c_{2} b_{3}=0$. Since $a_{3}\left(b_{4}-1\right)=-2 b_{3}, b_{4}-1 \neq 0$ and $a_{3}=2 r+1$, it follows that $b_{4}-1$ is even. The equation $c_{1}\left(1-b_{4}\right)+c_{2} b_{3}=0$ with $\left(c_{1}, c_{2}\right)=(-y r, y)$ now reads

$$
y r\left(b_{4}-1\right)+y \frac{-a_{3}\left(b_{4}-1\right)}{2}=y \frac{b_{4}-1}{2}[2 r-2 r-1]=0,
$$

which implies $y=0$.

For $a_{3} \geq-1$ and $a_{3}=2 r+1$, take $E=1$ and $D=v^{\left(b_{4}-1\right) / 2}$, where $v=d e^{-a_{3}} d$, and for $a_{3} \leq-2$ take $E=\left[d^{-1}, e-1\right]$ and $D=v^{\left(b_{4}-1\right) / 2}$, where $v=d e^{-a_{3}} d$, so in either case we have $E c D c^{-1} E^{-1} D=1$, therefore the equation admits the trivial solution.

(2c) If $a_{3}=2 r$ then a complete set of representatives of $E$ is given by elements of the form $(x, \bar{y})$, where $(x, y)$ are the coordinates relative to the base $((-1,0),(r,-1))$ and $\bar{y}$ means $y$ modulo 2 .

So a set of representatives consists of the elements of the form either $\left(c_{1}, c_{2}\right)=(-x, 0)$ or $\left(c_{1}, c_{2}\right)=(-x+r, 1)$.

From the necessary condition $c_{1}\left(1-b_{4}\right)+c_{2} b_{3}=0$ we conclude that if $E=(-x, 0)$ or $E=(-x+r,-1)$ then $x=0$. So, for $E$ and $D$ below, we have $E c D c^{-1} E^{-1} D=1$ and therefore the equation admits the trivial solution. 


\begin{tabular}{cccc}
\hline$a_{3}$ & $\left(c_{1}, c_{2}\right)$ & $E$ & $D$ \\
\hline $2 r \geq-1$ & $(0,0)$ & 1 & $\left(e^{-r} d\right)^{b_{4}-1}$ \\
$2 r \geq-1$ & $(-r, 1)$ & $e^{-r} d$ & $\left(d e^{-r}\right)^{b_{4}-1}$ \\
$2 r \leq-2$ & $(0,0)$ & {$\left[e^{-1}, d^{-1}\right]$} & $\left(e^{-r} d\right)^{b_{4}-1}$ \\
$2 r \leq-2$ & $(-r, 1)$ & $e^{-r} e^{-1} d e$ & $\left(d e^{-r}\right)^{b_{4}-1}$ \\
\hline
\end{tabular}

CAsE V: $A^{1}=\left(\begin{array}{cc}-1 & a_{3} \\ 0 & -1\end{array}\right), B^{1}=\left(\begin{array}{ll}1 & b_{3} \\ 0 & b_{4}\end{array}\right)$ and $a_{3}\left(b_{4}-1\right)=0$. The equation to be solved is

$$
Z_{3} E c Z_{2} c^{-1} E^{-1}\left(E\left(c D c^{-1} D\right) E^{-1}\left[E, D^{-1}\right]\right) D^{-1} Z_{3}^{-1} D D^{-1} Z_{2} D=1 .
$$

(1a) The necessary condition is

$$
2\left|Z_{2}\right|+\left|c D c^{-1} D\right|+\left|\left[E, D^{-1}\right]\right|=0 .
$$

Observe that $\left|c D c^{-1} D\right|$ depends on the choice of $D$, but $\left|\left[E, D^{-1}\right]\right|$ does not, since if $D_{1}=\alpha D$ and $E_{1}=\beta E$ with $\alpha, \beta \in \pi_{2}(T, T-1)$, then

$$
\left|c D_{1} c^{-1} D_{1}\right|=2|\alpha|+\left|c D c^{-1} D\right|
$$

and

$$
\left|\left[E_{1}, D_{1}^{-1}\right]\right|=\left|\left[E, D^{-1}\right]\right| .
$$

We conclude that the augmentation mod 2 , denoted by $\left.\right|_{2}$, is independent of $D$, and in order to calculate this condition we can take $D=e^{b_{3}} d^{b_{4}-1}$ and so $\left|c D c^{-1} D\right|_{2}+\left|\left[E, D^{-1}\right]\right|_{2}=0$ or

$$
b_{4}\left(b_{3}+1\right)+c_{1}\left(1-b_{4}\right)+c_{2} b_{3}-1 \equiv 0 \bmod 2 .
$$

(1b) The image of the map $\pi_{1}(T-1)=\langle e, d\rangle \rightarrow \mathbb{Z} \oplus \mathbb{Z}$ given by

$$
\alpha \mapsto\left(\left|\alpha c \alpha^{-1} c^{-1}\right|_{e},\left|\alpha c \alpha^{-1} c^{-1}\right|_{d}\right)
$$

is denoted by $\operatorname{im}\left(||_{e},\left.\right|_{d}\right)$. We have $e \mapsto(2,0), d \rightarrow\left(-a_{3}, 2\right)$.

(1c) If $a_{3}=2 r$, where $r \geq 0$, then

$$
\frac{\mathbb{Z} \oplus \mathbb{Z}}{\operatorname{im}\left(||_{e},||_{d}\right)}=\frac{\langle(1,0),(-r, 1)\rangle}{\langle 2(1,0), 2(-r, 1)\rangle} \cong \mathbb{Z}_{2} \oplus \mathbb{Z}_{2}
$$

Let $\left(|E|_{e},|E|_{d}\right)=\left(c_{1}, c_{2}\right)$. If $c_{2}$ is odd, then a set of representatives of $E$ is $\{(-r, 1),(-r+1,1)\}$. If $c_{2}$ is even, a set of representatives of $E$ is $\{(-2 r+1,2),(0,0)\}$ or $\{(1,0),(0,0)\}$.

(1d) If $a_{3}=2 r+1$ then

$$
\frac{\mathbb{Z} \oplus \mathbb{Z}}{\operatorname{im}\left(||_{e},\left.\right|_{d}\right)}=\frac{\mathbb{Z} \oplus \mathbb{Z}}{\langle 2(1,0),(-2 r-1,2)\rangle}=\frac{\langle(1,2),(0,1)\rangle}{\langle(1,2),(0,4)\rangle} \cong 0 \oplus \mathbb{Z}_{4} .
$$

Let $\left(|E|_{e},|E|_{d}\right)=\left(c_{1}, c_{2}\right)$. If $c_{2}$ is odd, a set of representatives of $E$ is $\{(0,3),(0,1)\}$ or $\{(1,1),(0,1)\}$. If $c_{2}$ is even, a set of representatives of $E$ is $\{(0,0),(0,2)\}$. 
(2a) For $a_{3}=2 r$, where $r \in \mathbb{Z}$, the sufficient condition is $\left(c_{1}, c_{2}\right) \not \equiv(0,0)$, that is, $c_{1} \not \equiv 0 \bmod 2$ or $c_{2} \not \equiv 0 \bmod 2$. For $a_{3}=2 r+1$, where $r \in \mathbb{Z}$, it is $\left(c_{1}, c_{2}\right) \not \equiv(0,0)$, that is, $c_{2} \not \equiv 0 \bmod 4$.

(2b) For $E$ and $D$ specified below we have $E c D c^{-1} E^{-1} D=1$, so the equation in (1) admits the trivial solution $Z_{2}=Z_{3}=1$ :

\begin{tabular}{cccrcc}
\hline$a_{3}=2 r>0$ & $c_{2}$ & $\left(b_{3}, b_{4}\right)$ & $\left(|E|_{e},|E|_{d}\right)=\left(c_{1}, c_{2}\right)$ & $E$ & $D$ \\
\hline$r$ even & odd & $\left(b_{3}, 1\right)$ & $(-r, 1) \equiv(0,1)$ & $d$ & $e^{b_{3}}$ \\
$r$ even & odd & $\left(b_{3}, 1\right)$ & $(-r+1,1) \equiv(1,1)$ & $e d$ & $e^{b_{3}}$ \\
$r$ odd & odd & $\left(b_{3}, 1\right)$ & $(-r+1,1) \equiv(0,1)$ & $d$ & $e^{b_{3}}$ \\
$r$ odd & odd & $\left(b_{3}, 1\right)$ & $(-r, 1) \equiv(1,1)$ & $e d$ & $e^{b_{3}}$ \\
& even & $(2 s, 1)$ & $(-2 r+1,2) \equiv(1,0)$ & $d e^{-2 r+1} d$ & $e^{s} d e^{s} d^{-1}$ \\
\hline
\end{tabular}

\begin{tabular}{cccrcc}
\hline$a_{3}=2 r<0$ & $c_{2}$ & $\left(b_{3}, b_{4}\right)$ & $\left(|E|_{e},|E|_{d}\right)=\left(c_{1}, c_{2}\right)$ & $E$ & $D$ \\
\hline$r$ even & odd & $\left(b_{3}, 1\right)$ & $(-r, 1) \equiv(0,1)$ & $d\left(d^{-1} e^{-1} d e\right)$ & $e^{b_{3}}$ \\
$r$ even & odd & $\left(b_{3}, 1\right)$ & $(-r+1,1) \equiv(1,1)$ & $e d\left(d^{-1} e^{-1} d e\right)$ & $e^{b_{3}}$ \\
$r$ odd & odd & $\left(b_{3}, 1\right)$ & $(-r+1,1) \equiv(0,1)$ & $d\left(d^{-1} e^{-1} d e\right)$ & $e^{b_{3}}$ \\
$r$ odd & odd & $\left(b_{3}, 1\right)$ & $(-r, 1) \equiv(1,1)$ & $e d\left(d^{-1} e^{-1} d e\right)$ & $e^{b_{3}}$ \\
& even & $(2 s, 1)$ & $(-2 r+1,2) \equiv(1,0)$ & $d e^{-2 r} d e$ & $e^{s} d e^{s} d^{-1}$ \\
\hline
\end{tabular}

\begin{tabular}{cccccc}
\hline$a_{3}=2 r+1>0$ & $c_{2}$ & $\left(b_{3}, b_{4}\right)$ & $\left(|E|_{e},|E|_{d}\right)=\left(c_{1}, c_{2}\right)$ & $E$ & $D$ \\
\hline & odd & $\left(b_{3}, 1\right)$ & $(0,3) \equiv(1,1)$ & $e d$ & $e^{b_{3}}$ \\
& odd & $\left(b_{3}, 1\right)$ & $(0,1) \equiv(0,1)$ & $d$ & $e^{b_{3}}$ \\
& even & $(2 s, 1)$ & $(0,2) \equiv(-2 r, 2)$ & $d e^{-2 r} d$ & $e^{s} d e^{s} d^{-1}$ \\
\hline
\end{tabular}

\begin{tabular}{rccrcc}
\hline$a_{3}=2 r+1<0$ & $c_{2}$ & $\left(b_{3}, b_{4}\right)$ & $\left(c_{1}, c_{2}\right)$ & $E$ & $D$ \\
\hline & odd & $\left(b_{3}, 1\right)$ & $(0,3) \equiv(1,1)$ & $e d\left(d^{-1} e^{-1} d e\right.$ & $e^{b_{3}}$ \\
& odd & $\left(b_{3}, 1\right)$ & $(0,1) \equiv(0,1)$ & $d\left(d^{-1} e^{-1} d e\right)$ & $e^{b_{3}}$ \\
& even & $(2 s, 1)$ & $(0,2) \equiv(-2 r, 2)$ & $d e^{-2 r-1} d e$ & $e^{s} d e^{s} d^{-1}$ \\
\hline
\end{tabular}

\begin{tabular}{ccccc}
\hline$a_{3}=0$ & $\left(c_{1}, c_{2}\right)$ & $\left(b_{3}, b_{4}\right)$ & $E$ & $D$ \\
\hline$(0,1)$ & $b_{3}$ odd, or & $e^{b_{3}-2} d e$ & $e^{b_{3}-1} d^{b_{4}-1} e$ \\
& & $b_{4}-1=2 k$ and $b_{3}$ even & $e^{-b_{3}+1} d e$ & $d^{k} e d^{k} e^{b_{3}-1}$ \\
& $(1,0)$ & $b_{4}-1=2 k+1$, or & $d^{2 k} e$ & $d^{2 k} e^{b_{3}} d$ \\
& $b_{4}-1=2 k$ and $b_{3}=2 s$ & $d^{-b_{4}+3} e$ & $e^{s} d e^{s} d^{2 k-1}$ \\
& $b_{3}=2 s$ or & $d e$ & $e^{s} d^{b_{4}-1} e^{s}$ \\
& $b_{4}-1=2 k$ & $d e$ & $d^{k} e^{b_{3}} d^{k}$ \\
\hline
\end{tabular}

(3) There is no solution for the remaining cases. To see this, consider $E$, $D$ as given below: 


\begin{tabular}{ccccc}
\hline$a_{3}$ & $\left(b_{3}, b_{4}\right)$ & $\left(c_{1}, c_{2}\right)$ & $E$ & $D$ \\
\hline $2 r>0$ & $(2 s, 1)$ & $\equiv(0,0)$ & 1 & $e^{2 s}$ \\
$2 r<0$ & $(2 s, 1)$ & $\equiv(0,0)$ & {$\left[d^{-1}, e^{-1}\right]$} & $e^{2 s}$ \\
$2 r+1>0$ & $(2 s, 1)$ & $\equiv(0,0)$ & 1 & $e^{2 s}$ \\
$2 r+1<0$ & $(2 s, 1)$ & $\equiv(0,0)$ & {$\left[d^{-1}, e^{-1}\right]$} & $e^{2 s}$ \\
0 & $(2 s, 2 k+1)$ & $\equiv(0,0)$ & 1 & $d^{k} e^{2 s} d^{k}$ \\
\hline
\end{tabular}

We write the term

$$
E c D c^{-1} D E^{-1}\left[E, D^{-1}\right]=E c D c^{-1} E^{-1} D
$$

in the generators $B_{(x, y)}$ of the abelianized group $\mathbb{Z} \pi_{1}(T)$.

For $a_{3} \neq 0$ we have $E c D c^{-1} E^{-1} D=\left[d^{-1}, e^{-2 s}\right]$, so

$$
\overline{E c D c^{-1} E^{-1} D}=\overline{\left[d^{-1},\left(e^{-1}\right)^{2 s}\right]}=B_{(-1,-1)}^{-1} B_{(-2,-1)}^{-1} B_{(-3,-1)}^{-1} \ldots B_{(-2 s,-1)}^{-1} .
$$

If $Z_{2}$ contains $B_{(x, y)}^{n}$ as a summand then

$$
E c Z_{2} c^{-1} E^{-1}=B_{\left(-x+a_{3} y+a_{3}-2,-y-2\right)}^{n}, \quad D^{-1} Z_{2} D=B_{(x-2 s, y)}^{n} .
$$

Let $H=\langle(2 s, 0)\rangle$ be the subgroup of $\pi=\pi_{1}(T) \cong \mathbb{Z} \oplus \mathbb{Z}$. Now in $\mathbb{Z}(\pi / H)$ the equation reduces to

$$
E c Z_{2} c^{-1} E^{-1} \overline{E c D c^{-1} E^{-1} D} D^{-1} Z_{2} D=1
$$

and

$$
\overline{E c D c^{-1} E^{-1} D}=\overline{\left[d^{-1},\left(e^{-1}\right)^{2 s}\right]}=B_{(2 s-1,-1)}^{-1} B_{(2 s-2,-1)}^{-1} B_{(2 s-3,-1)}^{-1} \ldots B_{(0,-1)}^{-1} .
$$

In order to cancel the term $B_{(s-1,-1)}^{-1}$ or $B_{(2 s-1,-1)}^{-1}$, either $B_{(s-1,-1)}$ or $B_{(2 s-1,-1)}$ must be a summand in $Z_{2}$. Then

$$
\begin{aligned}
E c Z_{2} c^{-1} E^{-1} & =B_{(s-1,-1)}^{1} \text { or } B_{(2 s-1,-1)}^{1}, \\
D^{-1} Z_{2} D & =B_{(s-1,-1)}^{1} \text { or } B_{(2 s-1,-1)}^{1} .
\end{aligned}
$$

So, the total exponent in $Z_{2}$ is even, therefore it is impossible to cancel $B_{(s-1,-1)}^{-1}$ or $B_{(2 s-1,-1)}^{-1}$.

For $a_{3}=0$, we have

$$
E c D c^{-1} E^{-1} D=c D c^{-1} D=\left[e^{-1} d^{-1}, d^{-k} e^{-2 s} d^{-k}\right]
$$

So

$$
\begin{aligned}
\left(c D c^{-1} D\right)^{-1} & =\left[d^{-k} e^{-2 s} d^{-k}, e^{-1} d^{-1}\right] \\
& =\left[d^{-k} e^{-2 s} d^{-k}, e^{-1}\right] e^{-1}\left[d^{-k} e^{-2 s} d^{-k}, d^{-1}\right] e
\end{aligned}
$$

and hence

$$
\begin{aligned}
c D c^{-1} D & =e^{-1}\left[d^{-1}, d^{-k} e^{-2 s} d^{-k}\right] e\left[e^{-1}, d^{-k} e^{-2 s} d^{-k}\right] \\
& =e^{-1} d^{-k}\left[d^{-1}, e^{-2 s}\right] d^{k} e\left[e^{-1}, d^{-k} e^{-2 s}\right] d^{-k} e^{-2 s}\left[e^{-1}, d^{-k}\right] e^{2 s} d^{k} \\
& =e^{-1} d^{-k}\left[d^{-1}, e^{-2 s}\right] d^{k} e\left[e^{-1}, d^{-k}\right] d^{-k} e^{-2 s}\left[e^{-1}, d^{-k}\right] e^{2 s} d^{k} .
\end{aligned}
$$


We now use the formula $\left[x, y^{n}\right]=[x, y] y[x, y] y^{-1} \ldots y^{n-1}[x, y] y^{-n+1}$ for the commutators $\left[d^{-1},\left(e^{-1}\right)^{2 s}\right]$ and $\left[e^{-1},\left(d^{-1}\right)^{k}\right]$ (here we suppose that $s>0$ and $k>0)$. We have

$$
\left[d^{-1},\left(e^{-1}\right)^{2 s}\right]=\left[d^{-1}, e^{-1}\right] e^{-1}\left[d^{-1}, e^{-1}\right] e \ldots e^{-2 s+1}\left[d^{-1}, e^{-1}\right] e^{2 s-1} .
$$

In $\mathbb{Z} \pi$ we have $\overline{\left[d^{-1}, e^{-1}\right]}=\overline{d^{-1} e^{-1} \operatorname{ded}^{-1} e^{-1}}=B_{(-1,-1)}^{-1}$ and therefore,

$$
\overline{\left[d^{-1},\left(e^{-1}\right)^{2 s}\right]}=B_{(-1,-1)}^{-1} B_{(-2,-1)}^{-1} B_{(-3,-1)}^{-1} \ldots B_{(-2 s,-1)}^{-1} \text {. }
$$

Next,

$$
\begin{aligned}
& {\left[e^{-1},\left(d^{-1}\right)^{k}\right]} \\
& \quad=\left[e^{-1}, d^{-1}\right] d^{-1}\left[e^{-1}, d^{-1}\right] d d^{-2}\left[e^{-1}, d^{-1}\right] d^{2} \ldots d^{-k+1}\left[e^{-1}, d^{-1}\right] d^{k+1} .
\end{aligned}
$$

In $\mathbb{Z} \pi$ we have

$$
\overline{\left[e^{-1}, d^{-1}\right]}=\overline{e^{-1} d^{-1} e d e^{-1} d^{-1} d e}=B_{(-1,-1)}
$$

and therefore,

$$
\overline{\left[e^{-1},\left(d^{-1}\right)^{k}\right]}=B_{(-1,-1)} B_{(-1,-2)} B_{(-1,-3)} \ldots B_{(-1,-k)} .
$$

Finally,

$$
\begin{aligned}
\overline{c D c^{-1} D}= & \left(B_{(-2,-1-k)}^{-1} B_{(-3,-1-k)}^{-1} \ldots B_{(-2 s-1,-1-k)}^{-1}\right) \\
& .\left(B_{(-1,-1)} B_{(-1,-2)} B_{(-1,-3)} \ldots B_{(-1,-k)}\right) \\
& .\left(B_{(-1-2 s,-1-k)} B_{(-1-2 s,-2-k)} B_{(-1-2 s,-3-k)} \ldots B_{(-1-2 s,-k-k)}\right) .
\end{aligned}
$$

Let $H=\langle(2 s, 0),(0,2 k)\rangle$ be the subgroup of $\pi \cong \mathbb{Z} \oplus \mathbb{Z}$. Now look at the equation in $\mathbb{Z}(\pi / H)$. The equivalence classes admit $B_{(x, y)}$ as a set of representatives for $0 \leq x \leq 2 s-1$ and $0 \leq y \leq 2 k-1$. After projecting on $\mathbb{Z}(\pi / H)$, we get

$$
\begin{aligned}
\overline{c D c^{-1} D}= & \left(B_{(2 s-2, k-1)}^{-1} B_{(2 s-3, k-1)}^{-1} \ldots B_{(0, k-1)}^{-1} B_{(2 s-1, k-1)}^{-1}\right) \\
& .\left(B_{(2 s-1,2 k-1)} B_{(2 s-1,2 k-2)} B_{(2 s-1,2 k-3)} \ldots B_{(2 s-1,2 k-k)}\right) \\
& .\left(B_{(2 s-1, k-1)} B_{(2 s-1, k-2)} B_{(2 s-1, k-3)} \ldots B_{(2 s-1,0)}\right) .
\end{aligned}
$$

The term $B_{(s-1, k-1)}^{-1} B_{(2 s-1,2 k-1)}$ is different from 0 since in this case $s \neq 0$ or $k \neq 0$ and it cannot be cancelled using the variable $Z_{2}$ (certainly also using the variable $\left.Z_{3}\right)$. In $\mathbb{Z}(\pi / H)$ this reduces to

$$
c Z_{2} c^{-1} \overline{c D c^{-1} D} D^{-1} Z_{2} D=1 \text {. }
$$

If $Z_{2}$ has the term $B_{(s-1, k-1)} B_{(2 s-1,2 k-1)}^{-1}$ then

$$
\begin{aligned}
c Z_{2} c^{-1} & =B_{(-s+1-2,-k+1-2)} B_{(-2 s+1-2,-2 k+1-2)}^{-1} \\
& =B_{(-s-1,-k-1)} B_{(-2 s-1,-2 k-1)}^{-1} .
\end{aligned}
$$


In $\mathbb{Z}(\pi / H)$ we get

$$
c Z_{2} c^{-1}=B_{(s-1, k-1)} B_{(2 s-1,2 k-1)}^{-1}
$$

and

$$
\begin{aligned}
D^{-1} Z_{2} D & =B_{(s-1-2 s, k-2 k-1)} B_{(2 s-2 s-1,2 k-2 k-1)}^{-1}=B_{(-s-1,-k-1)} B_{(-1,-1)}^{-1} \\
& =B_{(s-1, k-1)} B_{(2 s-1,2 k-1)}^{-1},
\end{aligned}
$$

which shows that we cannot make powers of $B_{(s-1, k-1)}$ and $B_{(2 s-1,2 k-1)}$ become zero.

CASE VI: $A^{1}=\left(\begin{array}{cc}-1 & a_{3} \\ 0 & 1\end{array}\right), B^{1}=\left(\begin{array}{ll}1 & b_{3} \\ 0 & b_{4}\end{array}\right)$ and $a_{3}\left(b_{4}-1\right)=2 b_{3}$ with $b_{4}-1 \neq 0$. The equation to be solved is

$$
Z_{3} E c Z_{2} c^{-1} E^{-1}\left(E\left(c D c^{-1} D^{-1}\right) E^{-1}[E, D]\right) D Z_{3}{ }^{-1} D^{-1} Z_{2}{ }^{-1}=1 .
$$

The augmentation homomorphism | | applied to the equation provides the condition

$$
-2\left|Z_{2}\right|+\left|c D c^{-1} D^{-1}\right|+|[E, D]|=0
$$

This condition modulo 2 is $\left|c D c^{-1} D^{-1}\right|_{2}+|[E, D]|_{2}=0 \bmod 2$.

We consider two cases: $a_{3}$ even and $a_{3}$ odd.

SuBCASE $a_{3}=2 r$ : Then $2 r\left(b_{4}-1\right)=2 b_{3}, A^{1}=\left(\begin{array}{cc}-1 & 2 r \\ 0 & 1\end{array}\right), B^{1}=\left(\begin{array}{cc}1 & r\left(b_{4}-1\right) \\ 0 & b_{4}\end{array}\right)$ with $b_{4}-1 \neq 0$. We summarize the data of this case by

$$
\left(A^{1}, B^{1},|E|_{e},|E|_{d}\right)=\left(\left(\begin{array}{cc}
-1 & 2 r \\
0 & 1
\end{array}\right),\left(\begin{array}{cc}
1 & r\left(b_{4}-1\right) \\
0 & b_{4}
\end{array}\right), c_{1}, c_{2}\right) .
$$

(1a) To calculate the necessary condition modulo 2 , take $v=e^{r} d$ so $c v c^{-1}=e^{-1} v e$. Now if $D=v^{b_{4}-1}$ then $c D c^{-1} D^{-1}=\left[e^{-1}, D\right]$ and therefore

$$
\begin{aligned}
\left|c D c^{-1} D^{-1}\right|_{2}+|[E, D]|_{2} & =\operatorname{det}\left(\begin{array}{cc}
-1 & r\left(b_{4}-1\right) \\
0 & b_{4}-1
\end{array}\right)+\operatorname{det}\left(\begin{array}{cc}
c_{1} & r\left(b_{4}-1\right) \\
c_{2} & b_{4}-1
\end{array}\right) \\
& =0 \bmod 2
\end{aligned}
$$

so

$$
\left(b_{4}-1\right)\left(c_{1}-1-c_{2} r\right)=0 \bmod 2 .
$$

(1b) To solve the equation for the input datum

$$
\left(A^{1}, B^{1},|E|_{e},|E|_{d}\right)=\left(\left(\begin{array}{cc}
-1 & 2 r \\
0 & 1
\end{array}\right),\left(\begin{array}{cc}
1 & r\left(b_{4}-1\right) \\
0 & b_{4}
\end{array}\right), c_{1}, c_{2}\right)
$$

is equivalent to solving for the input datum

$$
\left(A, B,|E|_{e},|E|_{d}\right)=\left(\left(\begin{array}{cc}
-1 & 0 \\
0 & 1
\end{array}\right),\left(\begin{array}{cc}
1 & 0 \\
0 & b_{4}
\end{array}\right), c_{1}-r c_{2}, c_{2}\right) .
$$


To see this, it is sufficient to consider the isomorphism $\varphi: G_{1} \rightarrow G_{2}$ given by

$$
e \mapsto e, \quad d \mapsto e^{-r} d, \quad c \mapsto c,
$$

where

$$
G_{1}=\left\langle e, d, c: c e c^{-1}=e^{-1}, c d c^{-1}=e^{-1} e^{2 r} d e\right\rangle
$$

is the group for the first datum, and

$$
G_{2}=\left\langle e, d, c: c e c^{-1}=e^{-1}, c d c^{-1}=e^{-1} d e\right\rangle
$$

is the group for the second datum.

(1c) Now we consider the input datum

$$
\left(A, B,|E|_{e},|E|_{d}\right)=\left(\left(\begin{array}{cc}
-1 & 0 \\
0 & 1
\end{array}\right),\left(\begin{array}{cc}
1 & 0 \\
0 & b_{4}
\end{array}\right), c_{1}-r c_{2}, c_{2}\right)
$$

satisfying the necessary condition

$$
\left(b_{4}-1\right)\left(c_{1}-1-c_{2} r\right)=0 \bmod 2 .
$$

In this case the map $\pi_{1}(T-1) \rightarrow \mathbb{Z} \oplus \mathbb{Z}$ given by

$$
\alpha \mapsto\left(\left|\alpha c \alpha^{-1} c^{-1}\right|_{e},\left|\alpha c \alpha^{-1} c^{-1}\right|_{d}\right)
$$

maps $e \mapsto(2,0)$ and $d \mapsto(0,0)$.

We consider the quotient group $\mathbb{Z} \oplus \mathbb{Z} /\langle(2,0)\rangle$. So, it suffices to take $E$ so that $\left(|E|_{e},|E|_{d}\right)=\left(0, c_{2}\right),\left(|E|_{e},|E|_{d}\right)=\left(1, c_{2}\right)$.

(2a) If $|E|_{e}=c_{1}-r c_{2} \equiv 1 \bmod 2$, then the problem has a solution. Take $D=d^{b_{4}-1}$ and $E=d^{c_{2}} e$ so $E c D c^{-1} E^{-1} D^{-1}=1$.

(2b) If $|E|_{e}=c_{1}-r c_{2} \equiv 0 \bmod 2$, then the necessary condition implies that $b_{4}-1$ is even, i.e. $b_{4}-1=2 k$. Let $L=\operatorname{gcd}\left(b_{4}-1, c_{2}\right)$. If $\left(b_{4}-1\right) / L=2 p$, then $c_{2} / L=2 q-1$ and in this case the equation has a solution. Take $v=c d c^{-1}=e^{-1} d e$ and note that $c v c^{-1}=d$. Now, if $D=\left(d^{L} v^{L}\right)^{p}$ and $E=v^{-L}\left(v^{L} d^{L}\right)^{q}$, then

$$
E c D c^{-1} E^{-1} D^{-1}=E\left(v^{L} d^{L}\right)^{p} E^{-1} D^{-1}=v^{-L}\left(v^{L} d^{L}\right)^{p} v^{L} D^{-1}=1 .
$$

(3) Now suppose $b_{4}-1=2^{n_{1}} p_{1}$, where $p_{1} \geq 1$ is odd, and $c_{2}=2^{n_{2}} p_{2}$, where $p_{2}$ is odd, with $1<n_{1} \leq n_{2}$ and $c_{1}-r c_{2} \equiv 0 \bmod 2$, where the last condition is equivalent to $c_{1} \equiv 0 \bmod 2$, since $c_{2}$ is even. Let us show that in this case we have no solution.

Let $D=d^{b_{4}-1}$ and $E=d^{c_{2}}$. Then

$$
\begin{aligned}
E\left(c D c^{-1} D^{-1}\right) E^{-1}[E, D]=E c D c^{-1} E^{-1} D^{-1} & =d^{c^{2}} c d^{b_{4}-1} c^{-1} d^{-c_{2}} d^{1-b_{4}} \\
& =d^{c_{2}} e^{-1} d^{b_{4}-1} e d^{-c_{2}} d^{1-b_{4}} \\
& =d^{c_{2}}\left[e^{-1}, d^{b_{4}-1}\right] d^{-c_{2}} .
\end{aligned}
$$


But since $\overline{\left[e^{-1}, d\right]}=B_{(-1,0)}^{-1}$ and $\overline{\left[e^{-1}, d^{-1}\right]}=B_{(-1,-1)}$ we have

$$
\overline{\left[e^{-1}, d^{b_{4}-1}\right]}= \begin{cases}B_{(-1,0)}^{-1} B_{(-1,1)}^{-1} B_{(-1,2)}^{-1} \ldots B_{\left(-1, b_{4}-2\right)}^{-1} & \text { if } b_{4}-1 \geq 1, \\ B_{(-1,-1)} B_{(-1,-2)} B_{(-1,-3)} \ldots B_{\left(-1,1-b_{4}\right)} & \text { if } b_{4}-1 \leq-1 .\end{cases}
$$

Therefore,

$$
\begin{aligned}
& \overline{E c D c^{-1} E^{-1} D^{-1}} \\
& \quad= \begin{cases}B_{\left(-1, c_{2}\right)}^{-1} B_{\left(-1,1+c_{2}\right)}^{-1} B_{\left(-1,2+c_{2}\right)}^{-1} \ldots B_{\left(-1, b_{4}-2+c_{2}\right)}^{-1} & \text { if } b_{4}-1 \geq 1, \\
B_{\left(-1,-1+c_{2}\right)} B_{\left(-1,-2+c_{2}\right)} B_{\left(-1,-3+c_{2}\right)} \ldots B_{\left(-1,1-b_{4}+c_{2}\right)} & \text { if } b_{4}-1 \leq-1 .\end{cases}
\end{aligned}
$$

The equation is

$$
Z_{3} E c Z_{2} c^{-1} E^{-1} \overline{E c D c^{-1} E^{-1} D^{-1}} D Z_{3}^{-1} D^{-1} Z_{2}^{-1}=1 .
$$

If we define $Z_{2}=B_{(x, y)}^{n}$ and $Z_{3}=B_{(z, w)}^{m}$ we have $c Z_{2} c^{-1}=B_{(-x-2, y)}^{-n}$, since $c B_{(0,0)} c^{-1}=B_{(-2,0)}^{-1}$. So, $E c Z_{2} c^{-1} E^{-1}=B_{\left(-x-2, y+c_{2}\right)}^{-n}$ and $D Z_{3}^{-1} D^{-1}$ $=B_{\left(z, w+b_{4}-1\right)}^{-m}$.

In fact, consider the subgroup $H=\langle(0, L)\rangle$ of $\mathbb{Z} \oplus \mathbb{Z}$, where $L=$ $\operatorname{gcd}\left(b_{4}-1, c_{2}\right)$ and $\left(b_{4}-1\right) / L=2 p+1$. Now, we look at the equation in $\mathbb{Z}(\pi / H)$. In $\mathbb{Z} \pi$ the equation

$$
Z_{3} E c Z_{2} c^{-1} E^{-1} \overline{E c D c^{-1} E^{-1} D^{-1}} D Z_{3}^{-1} D^{-1} Z_{2}^{-1}=1
$$

is given by

$$
\begin{aligned}
1=B_{(z, w)}^{m} B_{\left(-x-2, y+c_{2}\right)}^{-n} B_{\left(-1, c_{2}\right)}^{-1} B_{\left(-1,1+c_{2}\right)}^{-1} B_{\left(-1,2+c_{2}\right)}^{-1} & \ldots \\
\ldots & B_{\left(-1, b_{4}-2+c_{2}\right)}^{-1} B_{\left(z, w+b_{4}-1\right)}^{-m} B_{(x, y)}^{-n} .
\end{aligned}
$$

After projecting it on $\mathbb{Z}(\pi / H)$ we get

$$
B_{(-x-2, y)}^{-n} B_{(-1,0)}^{-2 p-1} B_{(-1,1)}^{-2 p-1} \cdots B_{(-1, L-1)}^{-2 p-1} B_{(x, y)}^{-n}=1 .
$$

In $\mathbb{Z}(\pi / H)$ we have $E c Z_{2} c^{-1} E^{-1}=B_{(-x-2, y)}^{-n}$. Therefore, the sum of the exponents of all $B_{(-1, i)}$ for $i=0, \ldots, L-1$ which appear in $E c Z_{2} c^{-1} E^{-1} Z_{2}^{-1}$ is even. On the other hand, this sum is $-2 p-1$, which is odd. So, there is no solution.

SubCASE $a_{3}=2 r+1$ : Then $b_{4}-1=2 k \neq 0$ and therefore $b_{3}=(2 r+1) k$,

$$
A^{1}=\left(\begin{array}{cc}
-1 & 2 r+1 \\
0 & 1
\end{array}\right), \quad B^{1}=\left(\begin{array}{cc}
1 & (2 r+1) k \\
0 & b_{4}
\end{array}\right), \quad a_{3}\left(b_{4}-1\right)=2 b_{3} .
$$

We summarize the input datum of this case by

$$
\left(A^{1}, B^{1},|E|_{e},|E|_{d}\right)=\left(\left(\begin{array}{cc}
-1 & 2 r+1 \\
0 & 1
\end{array}\right),\left(\begin{array}{cc}
1 & (2 r+1) k \\
0 & b_{4}
\end{array}\right), c_{1}, c_{2}\right) .
$$


(1a) To calculate the necessary condition modulo 2 , take $v=d e^{2 r+1} d$ so $c v c^{-1}=e^{-1} d^{-1} v d e$. Now, if $D=v^{k}$ then $c D c^{-1} D^{-1}=\left[e^{-1} d^{-1}, D\right]$ and therefore

$$
\begin{aligned}
\left|c D c^{-1} D^{-1}\right|_{2}+|[E, D]|_{2} & =\operatorname{det}\left(\begin{array}{cc}
-1 & (2 r+1) k \\
-1 & 2 k
\end{array}\right)+\operatorname{det}\left(\begin{array}{cc}
c_{1} & (2 r+1) k \\
c_{2} & 2 k
\end{array}\right) \\
& =0 \bmod 2
\end{aligned}
$$

so

$$
k\left(1+c_{2}\right)=0 \bmod 2 .
$$

(1b) To solve the equation for the input datum

$$
\left(A^{1}, B^{1},|E|_{e},|E|_{d}\right)=\left(\left(\begin{array}{cc}
-1 & 2 r+1 \\
0 & 1
\end{array}\right),\left(\begin{array}{cc}
1 & (2 r+1) k \\
0 & 2 k+1
\end{array}\right), c_{1}, c_{2}\right)
$$

is equivalent to solving it for the input datum

$$
\left(A, B,|E|_{e},|E|_{d}\right)=\left(\left(\begin{array}{cc}
-1 & 1 \\
0 & 1
\end{array}\right),\left(\begin{array}{cc}
1 & k \\
0 & 2 k+1
\end{array}\right), c_{1}-r c_{2}, c_{2}\right) .
$$

To see this it is sufficient to consider the isomorphism $\varphi: G_{1} \rightarrow G_{2}$ given by

$$
e \mapsto e, \quad d \mapsto e^{-r} d, \quad c \mapsto c,
$$

where

$$
G_{1}=\left\langle e, d, c: c e c^{-1}=e^{-1}, c d c^{-1}=e^{-1} e^{2 r+1} d e\right\rangle
$$

is the group for the first datum, and

$$
G_{2}=\left\langle e, d, c: c e c^{-1}=e^{-1}, c d c^{-1}=e^{-1} e d e\right\rangle
$$

is the group for the second datum.

(1c) Now, we consider the input datum

$$
\left(A, B,|E|_{e},|E|_{d}\right)=\left(\left(\begin{array}{cc}
-1 & 1 \\
0 & 1
\end{array}\right),\left(\begin{array}{cc}
1 & k \\
0 & 2 k+1
\end{array}\right), c_{1}-r c_{2}, c_{2}\right)
$$

satisfying the necessary condition

$$
k\left(1+c_{2}\right)=0 \bmod 2 .
$$

In this case the map $\pi_{1}(T-1) \rightarrow \mathbb{Z} \oplus \mathbb{Z}$ given by

$$
\alpha \mapsto\left(\left|\alpha c \alpha^{-1} c^{-1}\right|_{e},\left|\alpha c \alpha^{-1} c^{-1}\right|_{d}\right)
$$

maps $e \mapsto(2,0)$ and $d \mapsto(-1,0)$.

We consider the quotient group

$$
\frac{\mathbb{Z} \oplus \mathbb{Z}}{\langle(2,0),(-1,0)\rangle}=\frac{\mathbb{Z} \oplus \mathbb{Z}}{\langle(1,0)\rangle} .
$$

So, it suffices to take $E$ so that $\left(|E|_{e},|D|_{d}\right)=\left(a, c_{2}\right)$, where $a$ is fixed and $c_{2} \in \mathbb{Z}$.

(2a) If $|E|_{d}=c_{2} \equiv 1 \bmod 2$, i.e. $c_{2}=2 p-1$, then the problem has a solution. Take $D=(d e d)^{k}$ and $E=(d e d)^{p} d^{-1}$ so $E c D c^{-1} E^{-1} D^{-1}=1$. 
(2b) If $|E|_{e}=c_{2} \equiv 0 \bmod 2$, i.e. $c_{2}=2 p$, the necessary condition implies that $k$ is even. Let $L=\operatorname{gcd}\left(b_{4}-1, c_{2}\right)$; it is even as $c_{2}=2 p$. If $\left(b_{4}-1\right) / L=$ $2 p_{1}$ then $c_{2} / L=2 q+1$, and in this case the equation has a solution.

Indeed, first we observe that if $w_{1}=d e d$ and $w_{2}=c w_{1} c^{-1}=d d e=$ $d w_{1} d^{-1}$ then $c w_{2} c^{-1}=w_{1}$ so $c\left(w_{1} w_{2}\right)^{x} c^{-1}=\left(w_{2} w_{1}\right)^{x}$.

Now, if $D=\left(w_{1}^{L / 2} w_{2}^{L / 2}\right)^{p_{1}}$ and $E=w_{1}^{L / 2}\left(w_{2}^{L / 2} w_{1}^{L / 2}\right)^{q}$ then

$$
E c D c^{-1} E^{-1} D^{-1}=1 \text {. }
$$

(3) If $\left(b_{4}-1\right) / L=2 p+1$ and $c_{2}=2 q$ we are going to prove that the equation has no solution.

It is sufficient to prove that it has no solution for $D=w_{1}^{k}$ and $E=w_{2}^{q}$. We have

where

$$
E c D c^{-1} E^{-1} D^{-1}=w_{2}^{q} w_{2}^{k}\left(w_{2}^{q}\right)^{-1} w_{1}^{-k}=\left[d,(d e d)^{k}\right],
$$

$$
\overline{\left[d,(d e d)^{k}\right]}= \begin{cases}B_{(0,1)}^{-1} B_{(1,3)}^{-1} \ldots B_{(k-1,2 k-1)}^{-1} & \text { for } k \geq 1, \\ B_{(-1,-1)}^{-1} B_{(-2,-3)}^{-1} \ldots B_{(k, 2 k+1)}^{-1} & \text { for } k \leq-1 .\end{cases}
$$

The equation to be solved is

$$
Z_{3} E c Z_{2} c^{-1} E^{-1} \overline{E c D c^{-1} E^{-1} D^{-1}} D Z_{3}^{-1} D^{-1} Z_{2}^{-1}=1 .
$$

If $Z_{2}=B_{(x, y)}^{n}$ and $Z_{3}=B_{(z, w)}^{m}$ we obtain the following calculation for the terms of the above equation:

\begin{tabular}{ccccc}
\hline$Z_{3}$ & $E c Z_{2} c^{-1} E^{-1}$ & $\overline{E c D c^{-1} E^{-1} D^{-1}}$ & $D Z_{3}^{-1} D^{-1}$ & $Z_{2}^{-1}$ \\
$B_{(z, w)}^{m}$ & $B_{\left(-x-1+y+c_{2} / 2, y+c_{2}\right)}^{-n}$ & $\overline{\left[d,(d e d)^{k}\right]}$ & $B_{(z+k, w+2 k)}^{-m}$ & $B_{(x, y)}^{-n}$ \\
\hline
\end{tabular}

We consider the subgroup $H=\langle(1,0),(0, L)\rangle$ of $\mathbb{Z} \oplus \mathbb{Z}$, where $L=$ $\operatorname{gcd}\left(b_{4}-1, c_{2}\right)=\operatorname{gcd}(2 k, 2 q)$ and $2 k / L=\left(b_{4}-1\right) / L=2 p+1$. Now, we look at the equation in $\mathbb{Z}(\pi / H)$.

So, for $k \geq 1$, in terms of representative classes, the equation in $\mathbb{Z} \pi$ is $B_{(z, w)}^{m} B_{\left(-x-1+y+c_{2} / 2, y+c_{2}\right)}^{-n} B_{(0,1)}^{-1} B_{(1,3)}^{-1} \ldots B_{(k-1,2 k-1)}^{-1} B_{(z+k, w+2 k)}^{-m} B_{(x, y)}^{-n}=1$.

After projecting it on $\mathbb{Z}(\pi / H)$ we get

$$
B_{(x, y)}^{-n} B_{(0,1)}^{-(2 p+1)} B_{(1,3)}^{-(2 p+1)} \ldots B_{(L / 2-1,2 L / 2-1)}^{-(2 p+1)} B_{(x, y)}^{-n}=1 .
$$

In $\mathbb{Z}(\pi / H)$ we have $E c Z_{2} c^{-1} E^{-1}=B_{(x, y)}^{-n}$. Therefore, the sum of the exponents of all $B_{(i-1,2 i-1)}$, for $i=1, \ldots, L / 2$, which appear in $E c Z_{2} c^{-1} E^{-1} Z_{2}^{-1}$ is even. On the other hand, this sum is $-(2 p+1)$, which is odd. So, there is no solution.

We note that if $k \geq 1$ then, for all $i=1, \ldots, L / 2$, the $B_{(i-1,2 i-1)}$ are different classes in $\mathbb{Z}(\pi / H)$. In fact, if $1 \leq i<j \leq L / 2$ then $(j-1,2 j-1)-$ $(i-1,2 i-1)=\alpha(1,0)+\beta(0, L)$ and we do not have a solution in $\mathbb{Z}$ because $1 \leq j-i<L / 2$ so $L \nmid 2(j-i)$. 
If $k \leq-1$, the computation is the same, because in $\mathbb{Z}(\pi / H)$ we have $B_{(-1,-1)}^{-1} B_{(-2,-3)}^{-1} \ldots B_{(k, 2 k+1)}^{-1}=B_{(0,1)}^{-1} B_{(1,3)}^{-1} \ldots B_{(k-1,2 k-1)}^{-1}$.

Acknowledgments. We would like to thank the referee for his careful reading and comments, which helped to improve the manuscript.

\section{References}

[Ba-77] H. J. Baues, Obstruction Theory - on Homotopy Classification of Maps, Lecture Notes in Math. 628, Springer, 1977.

[Do-74] A. Dold, The fixed point index of fiber-preserving maps, Invent. Math. 25 (1974), 281-297.

[F-H-81] E. Fadell and S. Husseini, A fixed point theory for fiber-preserving maps, in: Lecture Notes in Math. 886, Springer, 1981, 49-72.

[F-L-P-79] A. Fathi, F. Laudenbach et V. Poénaru, Travaux de Thurston sur les surfaces, Astérisque 66-67 (1979).

[Go-87] D. L. Gonçalves, Fixed points of $S^{1}$-fibrations, Pacific J. Math. 129 (1987), 297-306.

[G-P-V-03] D. L. Gonçalves, D. Penteado and J. P. Vieira, The abelianized obstruction to fixed point free maps on torus bundles over $S^{1}$, in preparation.

[Ji-85] B. Jiang, Fixed points and braids II, Math. Ann. 272 (1985), 249-256.

[Ke-87] M. R. Kelly, Minimizing the number of fixed points for self-maps of compact surfaces, Pacific J. Math. 126 (1987), 81-123.

[Ni-27] J. Nielsen, Untersuchungen zur Topologie der geschlossenen zweiseitigen Flächen, Acta Math. 50 (1927), 189-358; English transl. in: Jakob Nielsen, Collected Mathematical Papers, Birkhäuser, 1986, 223-341.

[Pe-88] D. Penteado, Sobre pontos fixos de aplicações entre fibrados com fibra superfície, ICMSC-USP São Carlos-São Paulo, 1988.

[Pe-97] - Fixed points on surface fiber bundles, in: 10th Brazilian Topology Meeting, Mat. Contemp. 13 (1997), 251-267.

Departamento de Matemática

IME-USP

Caixa Postal 66.281

São Paulo 05311-970, Brazil

E-mail:dlgoncal@ime.usp.br

Departamento de Matemática

I.G.C.E-UNESP

Caixa Postal 178

Rio Claro 13500-230, Brazil

E-mail: jpvieira@rc.unesp.br
Departamento de Matemática Universidade Federal de São Carlos Rodovia Washington Luiz, Km 235 São Carlos 13565-905, Brazil E-mail: dirceu@dm.ufscar.br

Received 4 November 2003; in revised form 13 September 2004 